\title{
Specialized CFD Grid Generation Methods for Near-Field Sonic Boom Prediction
}

\author{
Michael A. Park, ${ }^{*}$ Richard L. Campbell, ${ }^{\dagger}$ and Alaa Elmiligui ${ }^{\ddagger}$ \\ NASA Langley Research Center, Hampton, VA 23681 \\ Susan E. Cliff ${ }^{\S}$ \\ NASA Ames Research Center, Moffett Field, CA 94035 \\ Sudheer N. Nayani $₫$ \\ Analytical Services \& Materials, Inc., Hampton, VA 23666
}

\begin{abstract}
Ongoing interest in analysis and design of low sonic boom supersonic transports requires accurate and efficient Computational Fluid Dynamics (CFD) tools. Specialized grid generation techniques are employed to predict near-field acoustic signatures of these configurations. A fundamental examination of grid properties is performed including grid alignment with flow characteristics and element type. The issues affecting the robustness of cylindrical surface extrusion are illustrated. This study will compare three methods in the extrusion family of grid generation methods that produce grids aligned with the freestream Mach angle. These methods are applied to configurations from the First AIAA Sonic Boom Prediction Workshop.
\end{abstract}

\section{Introduction}

There is interest in developing business jet ${ }^{1,2}$ and larger ${ }^{3,4}$ classes of supersonic vehicles. Supersonic flight over land is considered crucial to the commercial success of these designs but is currently limited in many countries, including the United States of America. The acceptance of an aircraft's sonic boom to the general population and regulatory agencies is a requirement for supersonic flights over land and therefore the commercial viability of a supersonic transport. Predicting how sonic boom signatures are perceived is a challenging task that requires the prediction of the signature on the ground. This task is complicated by long propagation distances, atmospheric variations, and the Earth's turbulent boundary layer. ${ }^{5}$ A detailed review of the history and state-of-the-art of sonic boom modeling is provided by Plotkin. ${ }^{6}$ Surveys of sonic boom prediction methods are available from $\mathrm{Ozcer}^{7}$ and Park. ${ }^{8}$

The propagation of a sonic boom is often separated into logical stages, or regions, to facilitate analysis. ${ }^{6}$ The near-field is a region near the aircraft where shocks are formed and are strongly influenced by nonlinear phenomena such as shock-shock interaction, shock curvature, and cross flow. Computational Fluid Dynamics (CFD) methods are typically employed in this near-field region. Sonic boom propagation methods ${ }^{6}$ are typically employed beyond the near-field where the geometric details of the configuration are less important than atmospheric variations and molecular relaxation phenomena. These atmospheric propagation methods rely on the availability of an accurate near-field signature.

Near-field CFD simulations often take advantage of the predictable characteristics and limited domains of influence of supersonic flow. There are many near-field CFD approaches and most of them include specialized techniques for grid generation and adaptation. A review of these previously published methods is provided in Section II, but there has been a limited number of comparisons between methods. The near-field CFD predictive capability of five methods was studied in an internal NASA workshop. ${ }^{9}$ The American Institute

\footnotetext{
*Research Scientist, Computational AeroSciences Branch, AIAA Senior Member.

${ }^{\dagger}$ Senior Research Engineer, Configuration Aerodynamics Branch, AIAA Associate Fellow.

${ }^{\ddagger}$ Research Aerospace Engineer, Configuration Aerodynamics Branch, AIAA Senior Member.

$\S$ Aerospace Engineer, Applied Modeling and Simulation Branch, AIAA Associate Fellow.

ฯSenior Scientist, CFD Group, 107 Research Drive, AIAA Senior Member.
} 
of Aeronautics and Astronautics (AIAA) is sponsoring the First AIAA Sonic Boom Prediction Workshop (SBPW-1 $)^{10,11}$ to examine methods employed by a broader section of the CFD community.

Three methods in the family of grid extrusion methods are examined in this article. These methods extrude the outer boundary of a "core" grid, in a series of steps or layers, to produce grid elements that align to the freestream Mach angle $\mu$,

$$
\mu=\sin ^{-1}(1 / M),
$$

where $M$ is Mach number. The extruded layers form a "collar" grid. These three methods will be applied to configurations from the SBPW-1 to document the properties of the methods. Sets of uniformly refined extruded grids were provided to the SBPW-1 for the two required cases. To introduce these extrusion methods, The effect of grid alignment on accuracy and the effect of extrusion surface topology on extrusion robustness are illustrated to indicate the key features of the extrusion process in Section III.

\section{Previous Work}

Cliff and Thomas ${ }^{12}$ used structured and isotropic unstructured grid methods without alignment to the freestream Mach angle. Djomehri and Erickson ${ }^{13}$ applied an adaptive isotropic unstructured grid FELISA $^{14,15}$ method. A linear acoustics propagation code was required to reach the validation data locations for these isotropic methods. Fouladi ${ }^{16}$ applied linear VGRID sources ${ }^{17}$ and USM3D ${ }^{18,19}$ to isotropic grids of a body of revolution. Choi, Alonso, and van der Weide ${ }^{20}$ applied isotropic grid adaptation in areas of large pressure gradient and produced good comparisons to distances less than one body length. Without alignment (using isotropic unstructured grids) only short distances were attempted or a propagation method was used to reach validation data locations. Carter and Deere ${ }^{21}$ examined the effect of unstructured grid resolution and showed a significant improvement with alignment of anisotropic grid to the freestream Mach angle. These anisotropic alignment methods were improved and evaluated by Campbell et al. ${ }^{22}$ Carter, Campbell, and Nayani ${ }^{23}$ examined a low boom configuration with laminar and turbulent analysis. These freestream Mach alignment methods evolved into the extrusion methods of Nayani and Campbell ${ }^{24}$ and Cliff et al. $^{25}$

Meredith et al. ${ }^{26}$ showed an overset grid system with Cartesian and freestream shock-aligned grids for the Shaped Sonic Boom Demonstrator (SSBD) vehicle. The shock-aligned grid provided better comparison to flight data measurements. Bui ${ }^{27}$ studied the grid resolution and alignment. He found that the Mach-aligned grid provided better prediction of cone-cylinder measurements than a Cartesian aligned grid, even at much higher spacial resolutions. Ishikawa et al. ${ }^{28}$ utilized structured grids inclined to Mach angle and examined the effect of grid resolution.

Siclari and Darden ${ }^{29}$ detailed efforts to align structured grids and adapt to shock locations. This adaptive approach was also employed by Page and Plotkin. ${ }^{30}$ Kandil et al. ${ }^{31,32}$ used various Euler methods very near the body. This near body solution was interpolated to an adaptively shock-aligned structured grid. Complete details of the shock-alignment method is provided by Ozcer ${ }^{7}$

Ozcer and Kandil ${ }^{33}$ applied FUN3D ${ }^{34,35}$ with an OptiGRID ${ }^{36}$ anisotropic grid adaptation method to resolve the pressure gradient by refining and aligning the tetrahedral grid. Alauzet and Loseille ${ }^{37}$ utilized anisotropic metric-based interpolation control of Mach number. The anisotropic adaptive method allowed second-order global mesh convergence in the $L_{2}$-norm of Mach field interpolation error. This framework was extended to goal-oriented adaptation by Loseille, Dervieux, and Alauzet. ${ }^{38}$ Jones, Nielsen, and Park ${ }^{39}$ adapted grids to a Mach number Hessian grid metric scaled with an off-body pressure integral output-based grid resolution request. The use of tetrahedral grid allowed arbitrary alignment to local flow characteristics. The output-based adaptation scheme was extended to tetrahedral cut-cells ${ }^{8}$ and viscous effects with frozen boundary layer grids. ${ }^{40}$ Barter ${ }^{41}$ applied an output-adaptive higher-order discontinuous Galerkin scheme with artificial viscosity to a laminar airfoil. Fidkowski and Darmofal ${ }^{42}$ developed a cut-cell scheme and adapted to an off-body pressure integral.

Nemec, Aftosmis, and Wintzer ${ }^{43}$ used Cart3 $\mathrm{D}^{44}$ along with adjoint-based mesh adaptation to predict the pressure signature of a diamond airfoil. Improvements due to grid alignment to freestream Mach angle, cost function formulation, and aspect ratio were examined. Cliff et al. ${ }^{45}$ illustrated the benefit of aligning a Cartesian direction with the freestream Mach angle. Aftosmis et al. ${ }^{46}$ showed that pressure signatures on the adapted grids at different off-track angles collapse to the same signature, as expected in axisymmetric flow.

Adaptation is important for complex flow, but a priori grid alignment has produced satisfactory results 
for problems with know characteristics. Alonso, Kroo, and Jameson ${ }^{47}$ generated structured H-mesh with grid lines canted at the freestream Mach angle to maximize the resolution of the pressure signature. The grid alignment has also been implemented by interpolating between multiple solvers or grid constructions. Laflin, Klausmeyer, and Chaffin ${ }^{48}$ used an adapted isotropic grid very near the model to facilitate grid generation. The NSU3D ${ }^{49}$ solver computed the solution in this region, which was interpolated to a structured mesh that is swept to align mesh cell faces with the freestream Mach lines and solved with CFL3D. ${ }^{50}$

Waithe $^{51}$ compared USM3D ${ }^{49}$ with generated isotropic and adapted anisotropic unstructured grids to OVERFLOW $^{52}$ with a freestream shock aligned overset grid. A hybrid method was employed that interpolates the unstructured solution to a cylinder for OVERFLOW initialization. Howe ${ }^{53}$ used Cart3D in combination with an OVERFLOW freestream shock aligned overset grid and examined how the location of interpolation surface affects the predicted signature. Ishikawa et al. ${ }^{54}$ utilized a hybrid method with an inner unstructured grid solution interpolated to a Mach angle aligned structured grid. This hybrid approach was also utilized by Morgenstern, Buonanno, and Marconi. ${ }^{55}$

\section{Grid Generation Considerations}

This article will extend previous efforts ${ }^{21,27,43,45}$ to examine the alignment of the grid to the flow features. Specifically, the previous works compared Cartesian aligned or isotropic unstructured grids to freestream Mach aligned grids. The current work investigates the degradation due to a small degree of misalignment that can result for complex flows around realistic configurations.

The issues related to extruding a cylindrical boundary introduced by Nayani and Campbell ${ }^{24}$ and Cliff et al. $^{25}$ is further investigated in this article. These methods extrude the outer boundary of a core grid to create a collar grid. Section III.C details the conditions that can result in inverted prismatic elements that invalidate the extruded collar grid and strategies to address this pitfall. Geometric growth is used by these methods to gradually increase the height of subsequent layers of this extraction.

\section{III.A. Geometric Growth}

A geometric growth of layer thickness is used by the extrusion methods. The first layer is typically constructed to approximately match cells sizes at the inner core grid and collar grid interface. The number of elements is reduced by increasing layer thickness and cell aspect ratio away from the model. Geometric growth produces a smooth variation of cell heights from the first layer to the last layer to accomplish these goals. The initial prism altitude or thickness $t_{0}$ and growth rate $r$ determines the thickness of layer $i$,

$$
t_{i}=t_{0} r^{i}
$$

where $i$ is $0, \ldots, n-1$ and $n$ is the total number of layers. The total thickness $T$ is given by,

$$
T=t_{0} \frac{1-r^{n}}{1-r},
$$

and Newton's method is used to solve for $r$ when $t_{0}, n$, and $T$ are specified.

\section{III.B. Grid Alignment}

A hexahedral grid consisting of a single span of cells is utilized to examine the impact of grid alignment on propagated shocks. This yields a $2 \mathrm{D}$ solution. The lower boundary is given by the expression,

$$
z(x)=\left\{\begin{array}{ll}
0 & x<0 \\
0.01 x & 0<=x<=1 \\
0.01 & 1<=x<=2 \\
0.01 x-0.01 & 2<=x<=3 \\
0.02 & 0<x
\end{array} .\right.
$$

These two ramps produce two pairs of oblique shocks and expansions as shown in Fig. 1. This combination of shocks and expansions is expected to transform from square wave into a sawtooth wave as the signal propagates, as seen in Fig. 2(a). The grid is extruded at the freestream Mach angle in Fig. 2(b) and has a constant spacing in the $x$-direction of $h_{x}=0.125$. The grid has a first cell height of $t_{0}=0.1$ and growth rate of $r=1.051$. The total height is $T=100$ after 80 layers. 


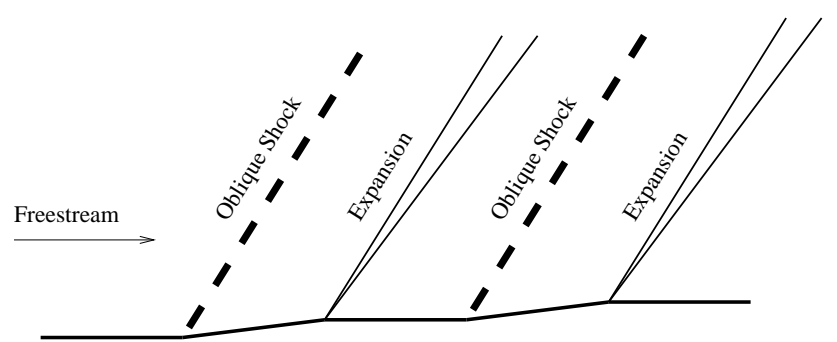

Figure 1. 2D solution diagram.

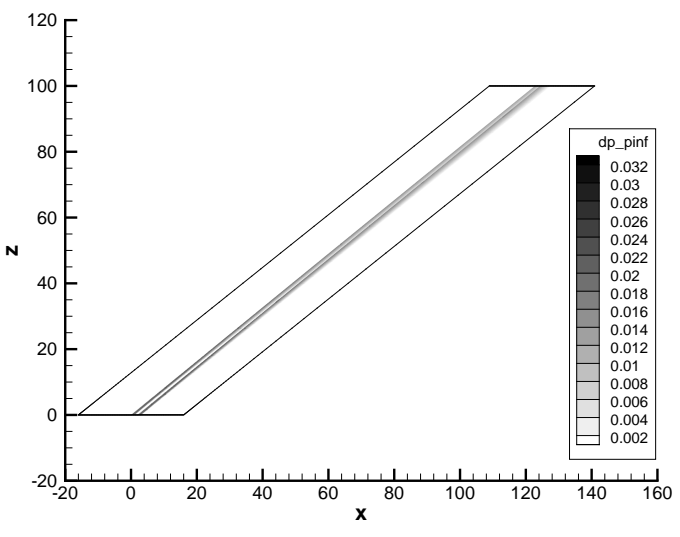

(a) Sheared domain and pressure disturbance.

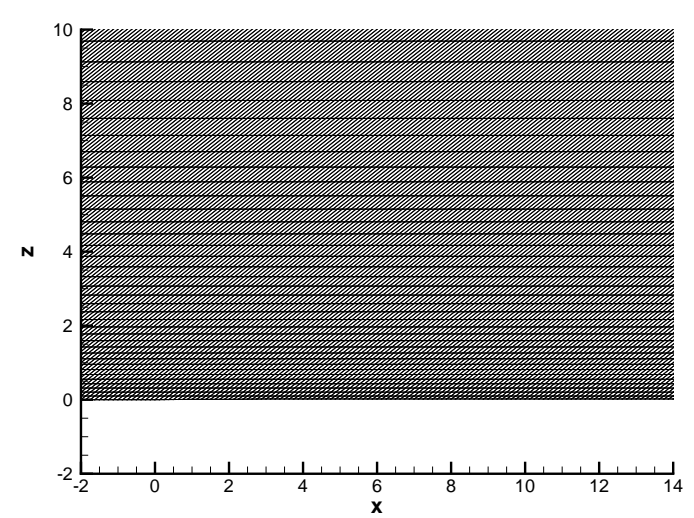

(b) Close-up of grid sheared at freestream Mach angle.

Figure 2. 2D sheared grids.

\section{III.B.1. Signature Propagation for Aligned and Misaligned Grids}

Two additional grids are created with the shear angle four degrees ahead and four degrees behind the freestream 1.6 Mach angle. The signatures produced by the three grids sheared ahead, at, and behind the freestream Mach angle are studied to observe the impact of grid alignment. The flux-vector splitting of van Leer ${ }^{56}$ is used with unlimited unweighted least-squares reconstruction as described by Anderson and Bonhaus. ${ }^{34}$ The pressure sampled at increasing propagation distance is shown in Fig. 3 for the three grids sheared ahead, at, and behind the freestream Mach angle. For $H=16.0$ ( $H$ is the displacement in the $z$-direction) and less the three grids produce very similar signatures as the pressure disturbance transforms from square waves to sawtooth waves, see Fig. 3(a-c). At $H=32.0$, a difference in the three shear angles is observed, see Fig. 3(d). The Mach angle aligned grid is producing the expected sawtooth signature and the misaligned grids are producing a smoothed signature. The signature of the grid that is inclined four degrees ahead of the Mach angle is more smooth than the signature of the grid that is inclined four degrees behind the Mach angle.

\section{III.B.2. Element Type and Refinement of Aligned and Misaligned Grids}

The effect of grid refinement and element type for Mach angle aligned and nonaligned grids at $H=64$ is shown in Fig. 4. The aligned grid exhibits the expected sawtooth signature for all configurations. The smooth signatures at $H=32.0$ of nonaligned grids is further smoothed at $H=64$. The two sawtooth waves have merged into a single smooth wave for the four degrees ahead of Mach angle grid, see Fig. 4(a). Converting the hex grid into a single layer of prisms also produces a single smooth signature for the four degrees behind of Mach angle grid in Fig. 4(b). Grid refinement is performed in the $x$ and $z$ directions. The geometric growth rate for the refined $z$ grid is $r=1.025$ to yield 160 layers with a total height of $T=100$. The $x$ refinement, shown in Fig. 4(c), does not significantly improve the sharpness of the nonaligned grids, but $z$ refinement, shown in Fig. 4(d), does improve the nonaligned grid signatures. The improvement with 


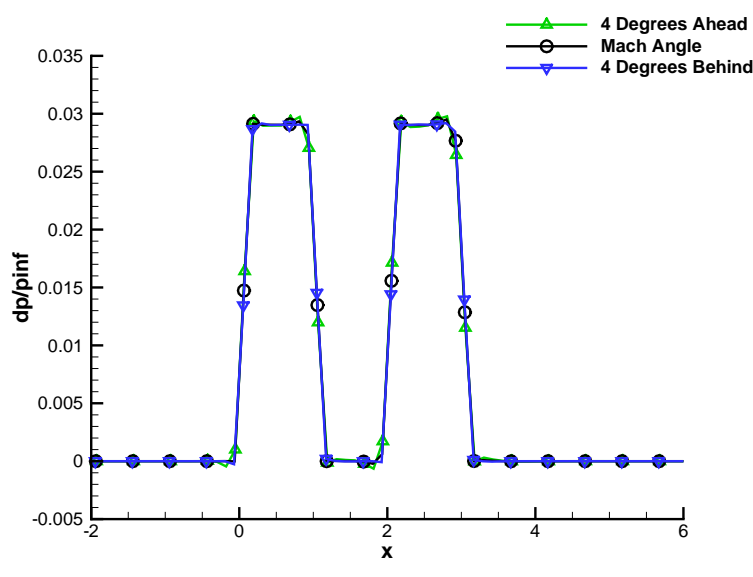

(a) Pressure at $H=0.05$.

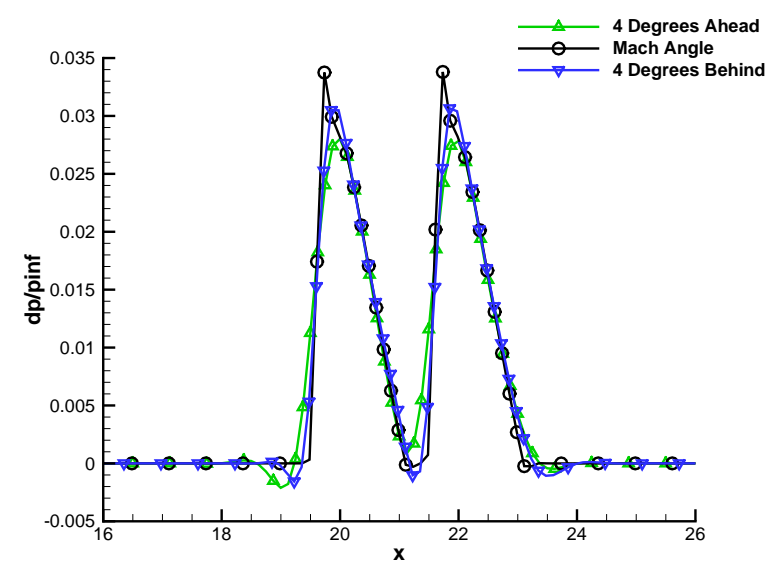

(c) Pressure at $H=16.0$.

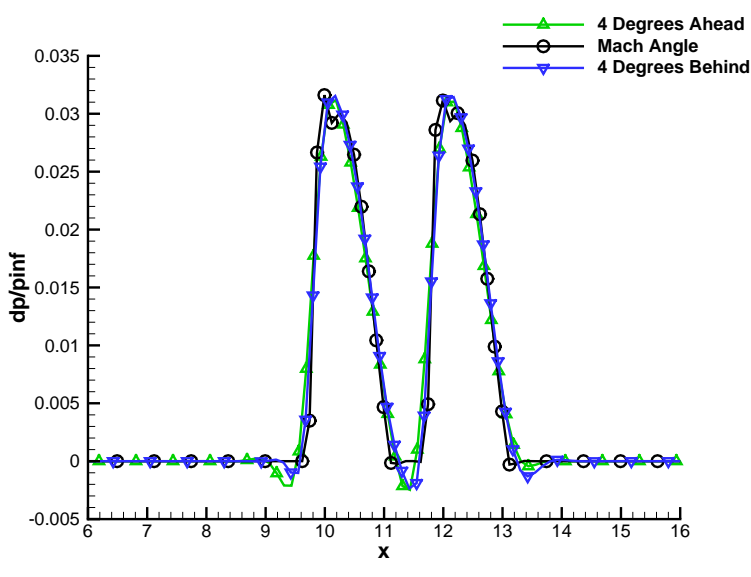

(b) Pressure at $H=8.0$.

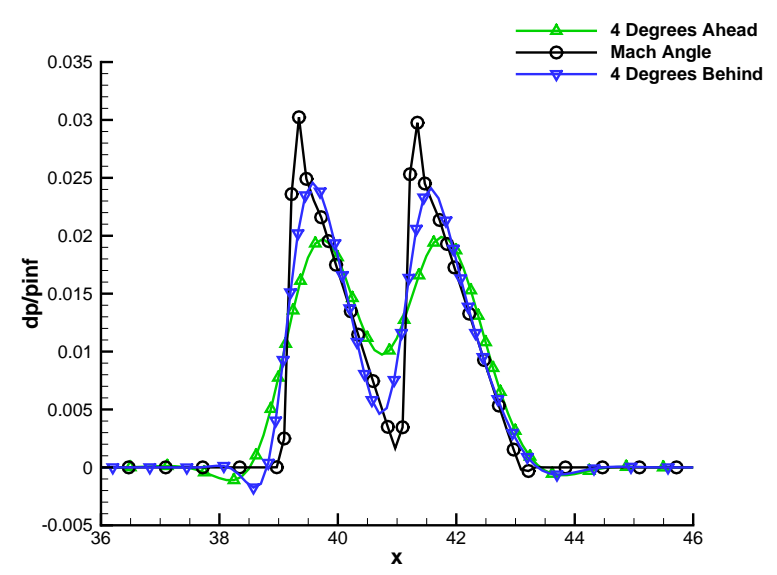

(d) Pressure at $H=32.0$.

Figure 3. 2D sheared grids. 
$z$ refinement may be connected to a reduction in cell aspect ratio for $z$ refinement, which appears to benefit the misaligned grids.

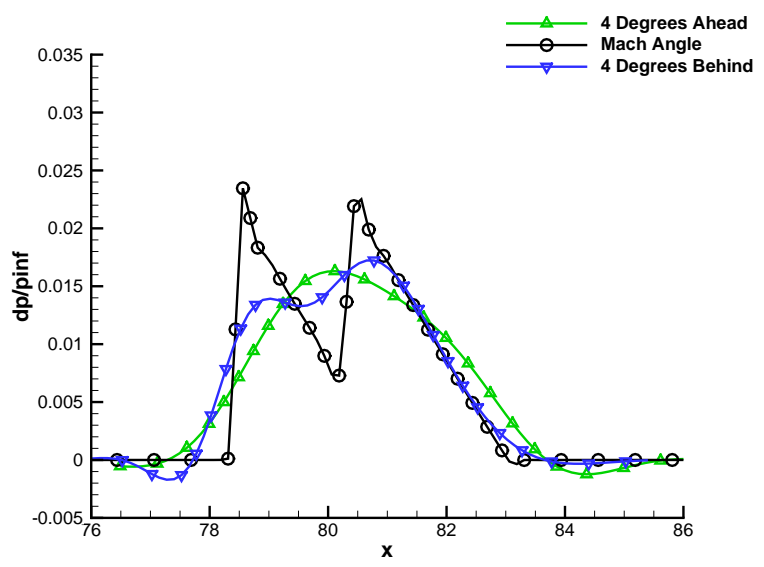

(a) Original spacing, hexes.

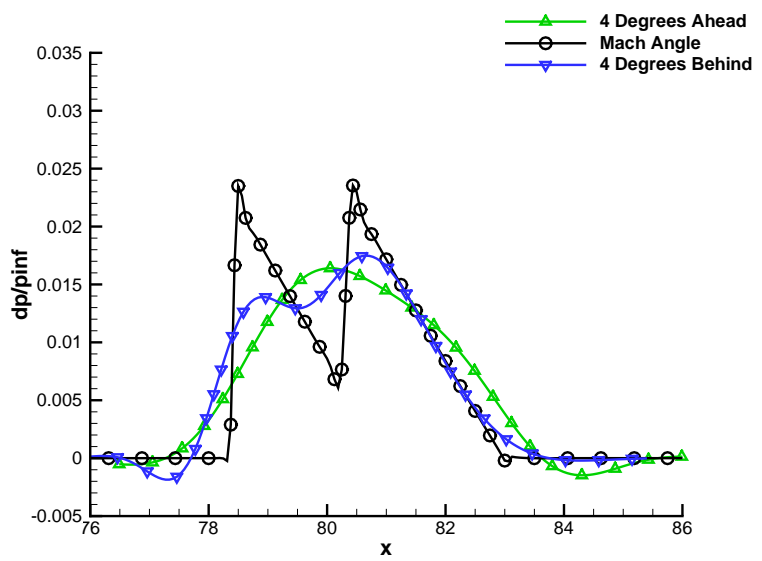

(c) Refinement in the $x$-direction, $0.5 h_{x}$.

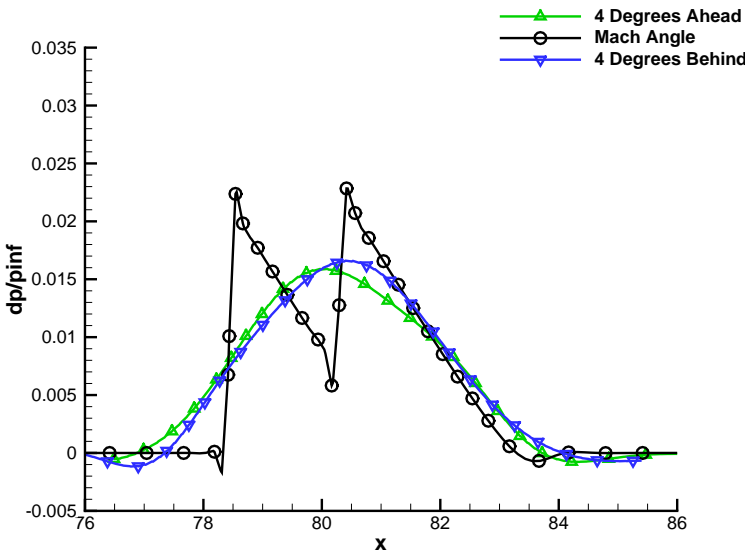

(b) Original spacing, prisms.

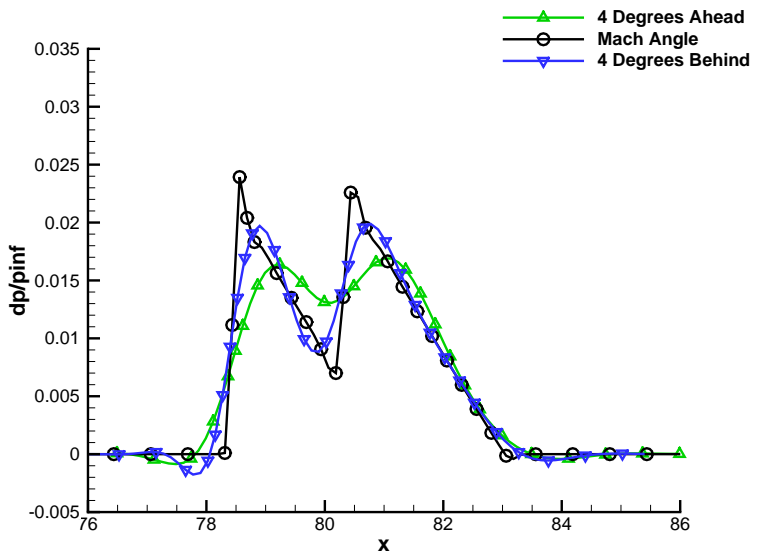

(d) Refinement in the $z$-direction, $0.5 h_{z}$.

Figure 4. Pressure at $H=64.0$.

\section{III.C. Cylindrical Surface Extrusion Pitfalls}

The three methods described in this article all extrude elements aligned with the freestream Mach angle from a triangulated surface. Even when the the nodes of this triangulation are projected to a cylinder, the normals of the linear triangles deviate from the analytical normal of the cylinder. If the dot-product of the triangle normal and extrusion direction becomes negative, an inverted prism is created that invalidates the grid.

Shewchuck ${ }^{57}$ examines the approximation of paraboloid surface normals by linear triangles. He illustrates that a triangulation with large angles have alternating normal directions like a "washboard." Poor approximation of a cylindrical surface produces surface normals that are not normal to the cylinder axis. These inclined triangles are used to form the bases of prism volumes that are extruded in the direction of freestream Mach angle. The radius of the cylindrical surface grows with successive extrusions and the triangles are elongated in the tangential direction. The elongation of a triangle with each node on a cylinder at different tangential locations will result in a decrease of the angle between the triangle's normal and the a cylinder axis. When the triangle's normal is inclined more than the freestream Mach angle, an extruded prism may invert.

Further study into the cause of the negative cells has shown that this is an inherent feature for grid 
extrusion methods if the core outer grid has surface triangles with a downstream point that does not line up directly behind one of the upstream points. The special case for a triangle with one side perpendicular to the cylinder axis is illustrated in Figure 5 . All 3 points of the triangle are on the outer cylinder of radius $R$ and point $\mathbf{c}$ is downstream of points $\mathbf{a}$ and $\mathbf{b}$ by a distance $d x$. Points $\mathbf{a}$ and $\mathbf{b}$ are offset from point $\mathbf{c}$ by $\mathbf{a}$ radial angle $\phi$. This offset results in a normal distance $d z$ which is given by

$$
d z=R(1-\cos (\phi))
$$

resulting in a face inclination angle $\lambda$ computed as

$$
\lambda=\tan ^{-1}(d z / d x)
$$

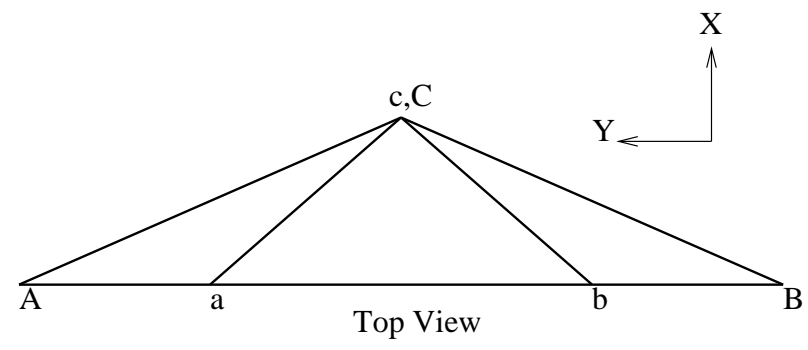

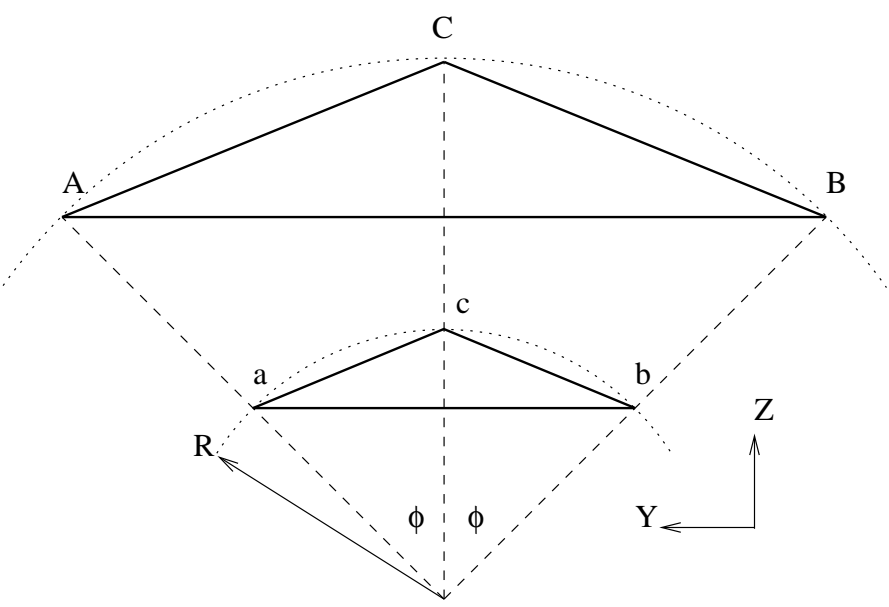

Front View
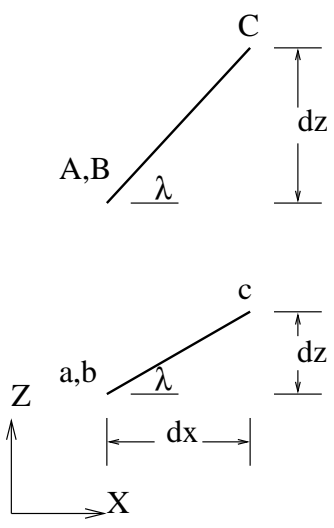

Side View

Figure 5. Illustration of non-aligned outer boundary face inclination problem.

As layers of cells are added to the collar grid, $d x$ remains fixed, but the value of $R$ increases, thus increasing $d y$ and $\lambda$. This is shown by the triangle with points $\mathbf{A}, \mathbf{B}$, and $\mathbf{C}$. The $\mathbf{A}, \mathbf{B}$, and $\mathbf{C}$ triangle is only twice the radius of the $\mathbf{a}, \mathbf{b}$, and $\mathbf{c}$ triangle and $\lambda$ has increased significantly in the "Side View." The $R$ can increase twentyfold in a typical application, which would increase $\lambda$ by a greater amount. Eventually, the boundary face inclination angle becomes parallel to the interior cell faces that are sheared at the Mach angle and the volume of the cell connected to that face becomes negative.

There are methods to control the angle between the surface normal and the cylinder axis. Arbitrary triangles with small angles will reduce this deviation, ${ }^{57}$ but not eliminate it. Constraining each extruded surface triangle so that one side is parallel to the cylinder axis will eliminate the error in the $x-r$ plane. A practical method to produce both triangles with an edge aligned to cylinder axis and maintain small angles is subdividing a structured grid, aligned to the curvature direction, into right triangles. A structured grid aligned to the curvature constrains variations in grid density. To allow a variation in grid density, a prism with a regular polygonal base can replace cylindrical surface. This creates an approximation to the cylinder with planar surfaces. The amount of approximation can be controlled by the number of polygonal sides. If the topology of this ruled surface between the polygonal bases is enforced during grid generation, the normal of planar surfaces is expressed exactly by any triangle regardless of shape and each triangle is exactly normal to the center axis by construction. Without constraints on the construction of the extrusion surface, refinement that splits long edges and can improve grids, but does not guarantee robustness. 


\section{Extrusion Methods}

Three methods are introduced to extrude the outer boundary of a core grid into layers that form a collar grid aligned to the freestream Mach angle. The geometric growth, grid alignment, and extrusion robustness discussions in the previous section are used to aid the description of these methods.

\section{IV.A. Boom Grid (BG) Generation Method}

The Boom Grid (BG) method ${ }^{24}$ is similar to other "extrusion" grid generation approaches in that the new grid is created by extending lines from grid points on a boundary surface of an existing grid in a direction approximately normal to the surface. The new cells are extruded through the outer, typically cylindrical, farfield boundary of an existing "core" 3D grid around a configuration, creating a "collar" grid, see Fig. 6. For triangular outer boundary faces on the core grid, the extruded cells are prisms. For methods that only support tetrahedra, each of these prisms is split into three tetrahedral cells. The BG method utilizes very efficient cell generation and splitting algorithms that require a few seconds to generate hundreds of millions of new tetrahedral collar grid cells.

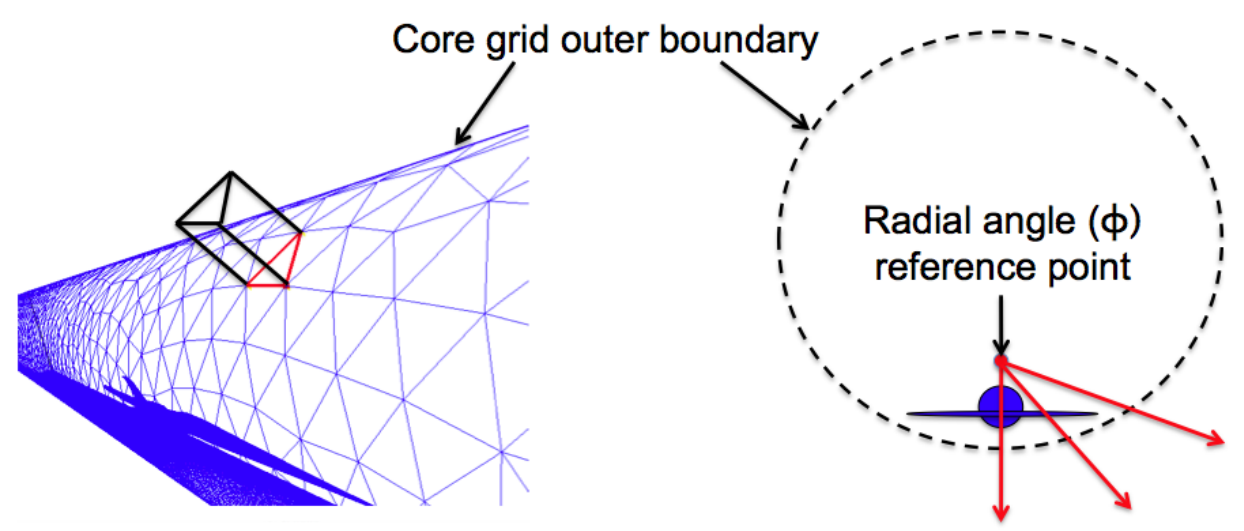

Figure 6. Prism extrusion process in the BG grid methodology.

The extension direction extends radially from the reference point when viewed from the front, see Fig. 6 . The extension direction is swept to the Mach angle when viewed from the side. This grid-line shearing, along with stretching of the distance between subsequent layers, creates high-aspect ratio cell faces that are closely aligned with the Mach angle so that the numerical dissipation of the boom signature is reduced. As few as 20 or more than 100 layers are generated to reach the desired location for extracting a sonic boom signature.

If the outer boundary is a cylinder and the collar grid lines are extruded normal to the original core grid outer boundary, then all of the extruded grid lines appear to originate at the center of the circle (front view of the cylinder) as a radial reference point. If the core grid has the configuration located at this reference point, then boom signature propagation lines will be approximately aligned with the radial grid lines, as desired. The weakness of this approach, however, is that there can be a significant distance from the bottom of the fuselage to the beginning of the collar grid. As the grid is not sheared and stretched in this core grid region, the signal can be dissipated before reaching the collar grid.

If only on-track signatures are needed, then the above problem can be resolved (though with some addition cost due to more grid cells) by simply increasing the radius of the outer core grid cylinder to allow the configuration to be shifted down closer to the lower boundary. For off-track signatures, however, this approach gives poor alignment of the grid lines and signal propagation direction. The BG method attempts to address this problem by using a radial reference point that is not the center of the circle. This allows the configuration to be shifted down closer to the outer boundary and still have the radial lines that emanate from the configuration for better signal propagation. The radial reference point can be located to focus the grid lines in a desired off-track direction to provide better signal resolution at the off-track angle with very little degradation of the on-track signature. Figure 7 illustrates this capability, showing collar grid inflow 
planes for standard on-track and focused off-track cases. Nayani and Campbell ${ }^{24}$ provides more detail on this option.

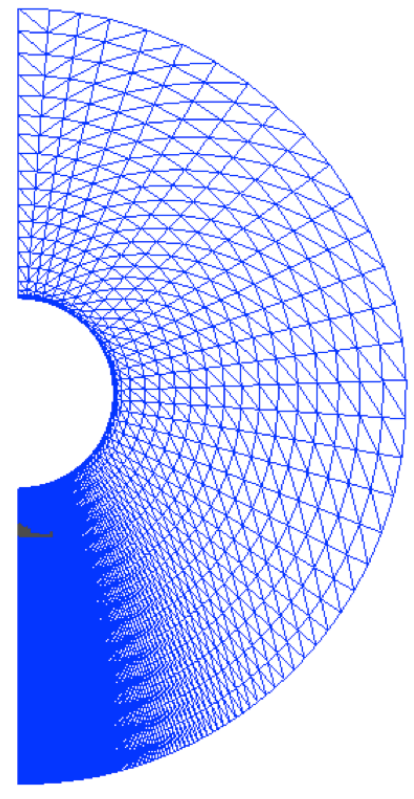

(a) On-track.

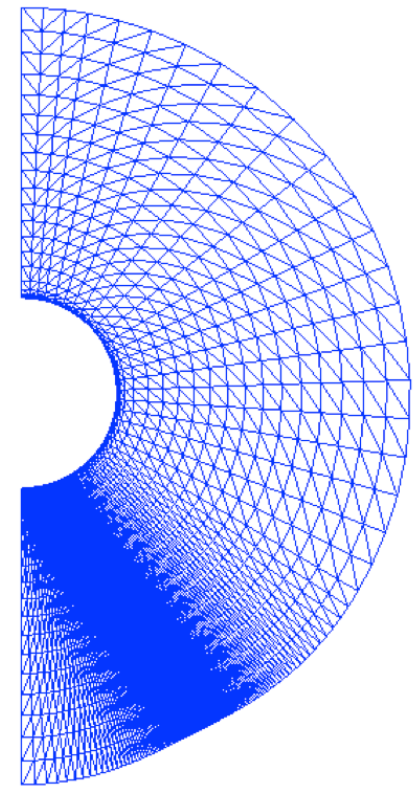

(b) Off-track.

Figure 7. Effect of BG grid-focusing option for off-track analysis on collar grid inflow plane.

As noted by Nayani and Campbell, ${ }^{24}$ the BG method is an improvement over the previous SSGRID ${ }^{22,58}$ and $\mathrm{SSG}^{24}$ grid stretching and shearing codes in terms of being able to reach a given distance below the aircraft before creating negative volume cells. BG has successfully generated collar grids with over 1,400 cell layers to distances of 100 body lengths without negative cells when the original core grid met the requirement of every face having one edge parallel to the cylindrical outer boundary axis. An automated method for creating an input file for the VGRID grid generation method that enforces this requirement has been developed and is referred to as the Cylindrical Outer Boundary (COB) code. In addition to achieving large signature location distances, the use of $\mathrm{COB}$ also allows the $\mathrm{BG}$ code to successfully obtain grids focused at off-track angles up to 90 degrees.

\section{IV.B. Mach Cone Aligned Prism (MCAP) Generation Method}

The Mach Cone Aligned Prism (MCAP) collar grid approach was developed by Cliff et al. ${ }^{25}$ and applied the to Delta Wing Body and Low-Boom Wing Body Tail configurations. Elmiligui et al. ${ }^{59}$ applied MCAP to a wing body configuration. MCAP extrudes the triangular faces of the cylindrical outer boundaries of a core tetrahedral mesh into a prismatic collar grid where the prisms are subdivided into tetrahedra. The shearing angle of the prism cells is adjusted for the angle of attack and Mach angle to allow for both on- and off-track aligned grids. These prisms are subdivided into tetrahedra to produce a grid composed entirely of tetrahedral cells. This provides an automated process to construct grids suited to obtain accurate sonic boom pressure signatures. Thus, configurations with any level of geometric complexity can be analyzed, provided a tetrahedral near-body volume grid of tetrahedra with a cylindrical boundary is supplied.

The core near-body grids may be constructed in a manner that is suitable for either viscous or inviscid computations. The radius of the cylinder should be small so that the boundaries are close enough to the vehicle to allow the Mach cone aligned prism cells to begin near the body before signals are dissipated by the near-body tetrahedra. An example of a cylindrically-shaped mesh and configuration is shown in Fig. 8. The fore and aft boundary faces and symmetry plane are removed in the figure for clarity. These fore and aft boundaries can be flat or aligned with the Mach angle. Shearing the aft boundary to form an inverted cone shape is an effective technique to remove unnecessary points downstream of the model.

The mesh density within the cylindrical grid needs to be sufficiently dense within the sonic boom region of influence so that the pressure signature can be taken far from the model, and configuration variations in a 


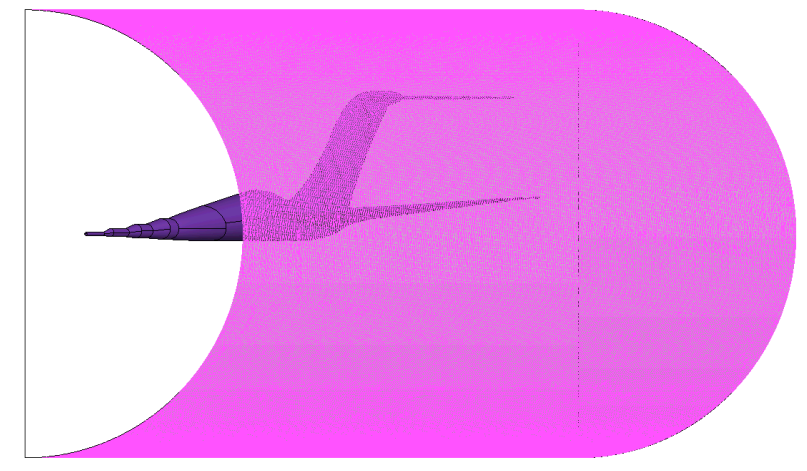

Figure 8. Cylindrical Mesh.

design environment would not require different volume grid densities. The grid will need to be highly refined within the Mach cone and behind the model for approximately a third to a half body length to capture details of the bow, tail, and return to ambient pressure signatures. Typically, refinement is necessary up to 90 degrees off-track even for on-track computations. To produce a high-density collar grid, a refined triangular cylinder grid is required.

MCAP inherits the grid spacing in the axial direction from the cylinder triangulation, and adjusts the radial stretching and shearing to align with the Mach cone angle around the aircraft. Each triangular face is extruded to form a prism that is sheared to align with the Mach angle. Each prism is split into three tetrahedra, which adds an edge to each of the quadrilateral faces of the prism cells. The quadrilateral face of the prism may not be a parallelogram since the extruded triangle edge is larger in the tangential direction than the previous layer due to the increasing radius of the cylinder. A prism and the three tetrahedra from splitting the prism are shown in Fig. 9. The topology of the subdivision of each prism into three tetrahedra is the same for all layers, as shown in a shaded and exploded view in Fig. 9(b).

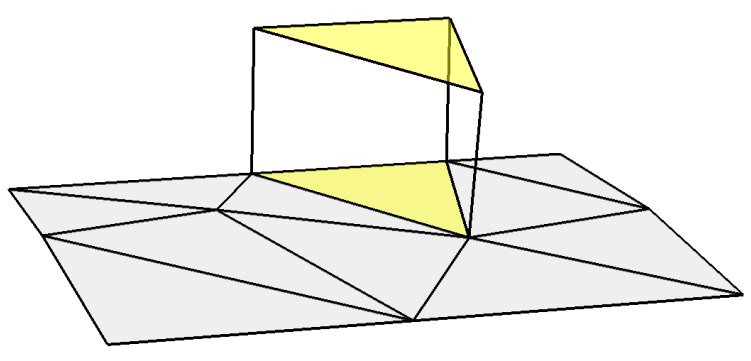

(a) Extruded prism.

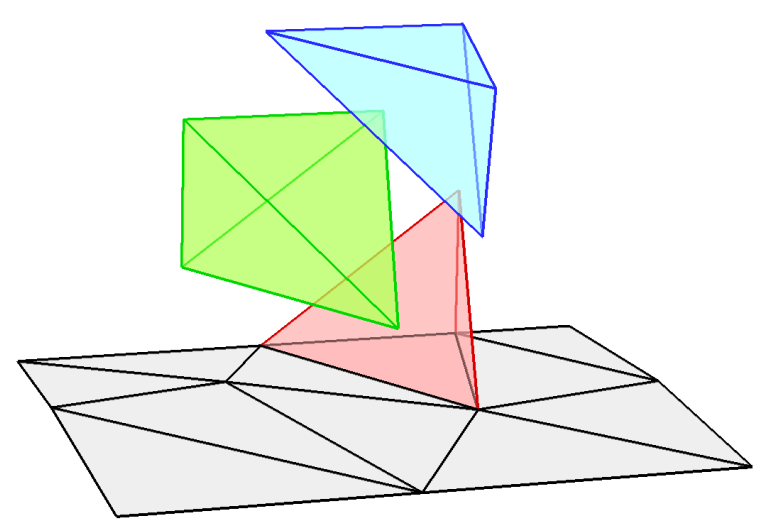

(b) Subdivision into tetrahedra (exploded view).

Figure 9. Prism extrusion and subdivision.

The extruded cylindrical mesh is sheared downstream at each layer for alignment of the mesh with the freestream Mach cone at angle $\mu$. Each mesh point is shifted by

$$
d x=\frac{t}{\tan (\nu)},
$$

where $d x$ is added to the $x$ coordinate shown in Fig. 10 and $t$ is the thickness of the layer in the radial direction. When the angle of attack is zero, $\nu=\mu$, which results in a symmetric shear. The thickness for 
each successive layer can grow geometrically, as shown in Fig. 10, or be held constant if the growth ratio is set to one.

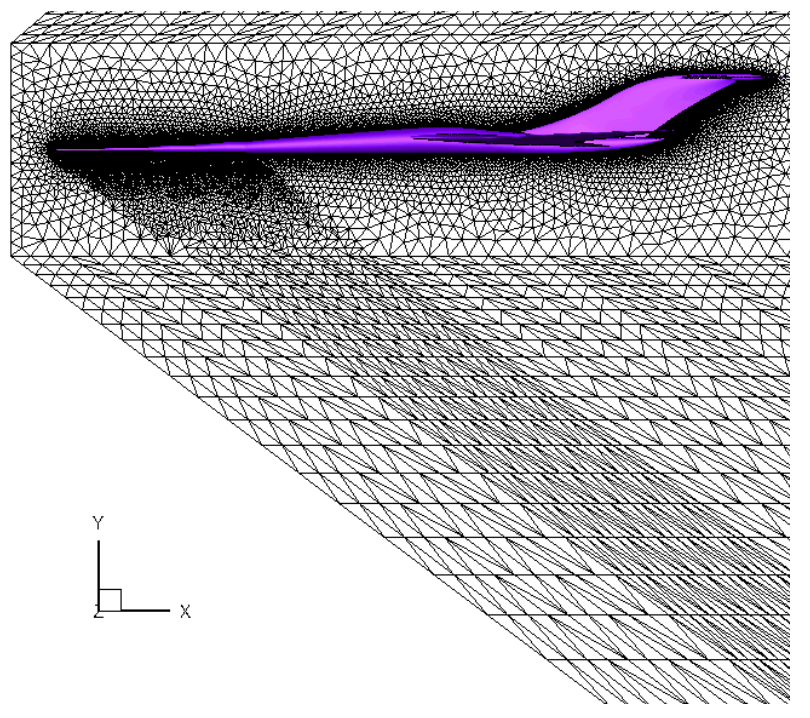

Figure 10. Core and extruded collar symmetry plane grid and model.

When the angle of attack $\alpha$ is not zero, then an additional asymmetrical shear is applied that depends on $\alpha$ and $\phi$, where $\phi=\tan ^{-1}(-z / y)$. A periodic weighting of $\alpha$ is given by

$$
\sigma=\frac{1-\cos (\phi)}{2}
$$

and the shear angle becomes

$$
\nu=\mu+(-\alpha(1-\sigma)+\alpha \sigma) .
$$

The weighting function is an approximation to the intersection of an $\alpha$-rotated cone and a cylinder where the intersection is an ellipse in the $\alpha$-rotated plane. This approximation is sufficient for supersonic vehicles, where angles of attack are expected to be small.

With each additional prism layer there is increased stretching of the mesh in the tangential direction due to the growth of the radius to the outer boundary. Thus, in order to obtain accurate solutions at large distances from an aircraft the stretched triangles and tetrahedra should be split to improve tangential refinement of the mesh. Mesh splitting should only be used when necessary because it approximately doubles the mesh point count and triples the cell count on the split layer and all successive prism layers.

The highly stretched tetrahedra on the outer prism layers have faces with two very long edges and one short edge. Thus, in order to reduce the tangential stretching of the prism mesh, the MCAP software allows the user to split the tetrahedra of one or more prism layers during the construction of the prismatic mesh. The algorithm splits only the longest two edges of each face on the outer boundary and the tetrahedra associated with this refined face. Splitting all edges would have been less difficult to program but would not have alleviated the stretching or improved the quality of the mesh.

Splitting amounts to adding data to lists of points, faces, and tetrahedra. The algorithm loops over the indices of the face elements on the outer cylinder. Each face is typically split into three faces with the same connectivity as shown in Fig. 11. The labeled points as described in the figure text can be found regardless of orientation. The algorithm determines whether the edges of neighboring faces had been split before splitting. Therefore if a small edge of a previous split triangle is common with the mid-length or long edge of the current face, then that edge will not be split. Thus it is possible to not split the face or split it into 2 to 4 triangles. There are a finite number of conditions encountered and the connectivity of face points is defined depending on the condition.

The tetrahedra associated with the split faces must be split as well. This involves two searches of the tetrahedron list. First, search for the tetrahedron with three common points of the face being split. The new tetrahedra are simply the faces plus the fourth point of the un-split tetrahedron illustrated by dashed edges in Fig. 11. The second search is to find any tetrahedra that contain the fourth point and two points of the 


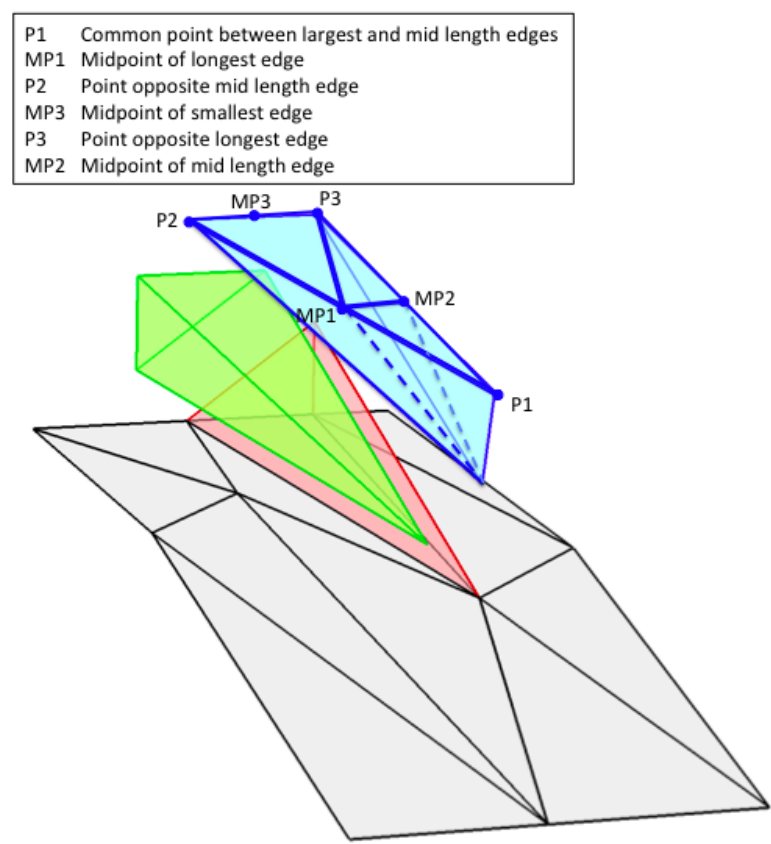

Figure 11. Details of extrusion layer refinement.

face being split, shown by the center medium gray cell in the example. If the edge of the abutting triangle is split then the neighboring tetrahedron must be split. This is not the case in this example, but would be if the upper dark gray tetrahedral were split at MP3. These two searches are typically non-exhaustive since they terminate when the tetrahedron is found. Each face and associated tetrahedron are split until the face list on the outer cylinder is exhausted. The program then copies the refined layer connectivity for the remaining layers of prismatic cells. Each newly added point is adjusted radially so that it is the same distance from the cylinder axis as its neighbors. This adjustment reduces the deviation between the triangle normal and the normal of a cylindrical surface by reducing $d y$ in Fig. 5 .

\section{IV.C. Inflate Generation Method}

The Inflate collar grid generation method can utilize a core grid with a triangular surface from any source. To guarantee robustness, the outer core grid surface should be composed of planar surfaces parallel to the cylinder axis. A prism with regular polygonal bases satisfies this constraint and the sides of the regular polygons can be increased to improve the cylindrical approximation of the ruled surfaces.

To form a layer, each node in the current triangular surface is first extruded in the radial direction $\mathbf{d}$ a distance $l$ so that the dot product of the displacement vector and the adjacent triangle normals is the requested thickness $t_{i}$,

$$
\begin{gathered}
l=\hat{\mathbf{d}} \mathbf{r} \cdot \hat{\mathbf{n}}, \\
\mathbf{d}=\frac{t_{i}}{l} \hat{\mathbf{d}} \mathbf{r}+\frac{t_{i}}{\tan (\nu)} \hat{\mathbf{d}} \mathbf{x},
\end{gathered}
$$

where $\hat{\mathbf{n}}$ a unit triangle normal, $\hat{\mathbf{d r}}$ is a unit vector in the radial direction, and $\hat{\mathbf{d x}}$ is a unit vector in the positive $x$ direction. Calculating the extrusion distance of each node maintains planar surfaces in each successive layer. This is accomplished through Eq. (11) and Eq. (10) where the extrusion distance is increased as the adjacent triangle normals deviate from the analytic normal of a cylindrical surface.

An example of a topology with regular polygonal outer boundary is shown in Fig. 12(a). It is a quarter domain of with a cone cylinder in the lower right portion of Fig. 12(a). Symmetry planes for the bottom and right of Fig. 12(a). The outer boundary in the upper left is composed of ruled surfaces connecting the sections of regular polygons at either end. In this example, four sides are shown of the twelve sides that compose the entire polygon. The outer boundary is a poor approximation of a cylinder in this example, but the number of sides can be arbitrarily increased to reduce the approximation. Each of these ruled surfaces is extruded to create the topology in Fig. 12(b). 


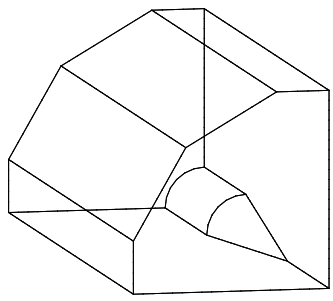

(a) Core grid topology.

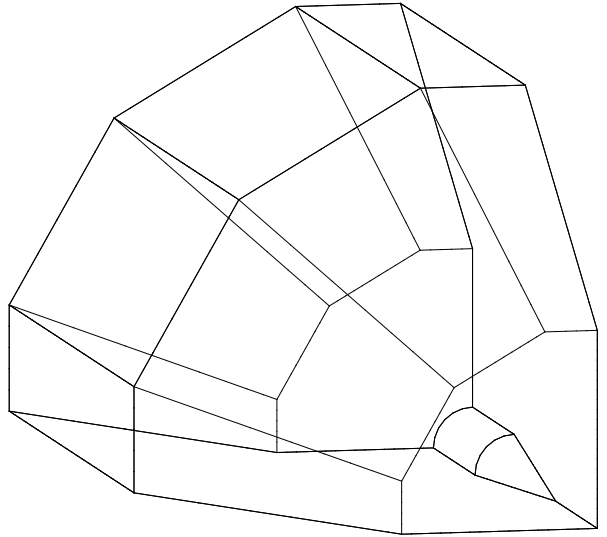

(b) Core and inflated collar grid topology.

Figure 12. Topology of a quarter of a cone-cylinder model domain.

The surface grids of the typologies shown in Fig. 12 are shown in Fig. 13. The core grid in Fig. 13(a) is composed of tetrahedra and the outer surfaces are composed of triangles with arbitrarily resolution and orientation. Each outer surface triangle in Fig. 13(a) is extruded into a prism in the same fashion as Fig. 9(a). The quadrilateral sides of the prisms are seen in Fig. 13(b) and each layer remains parallel to the original core grid outer surfaces.

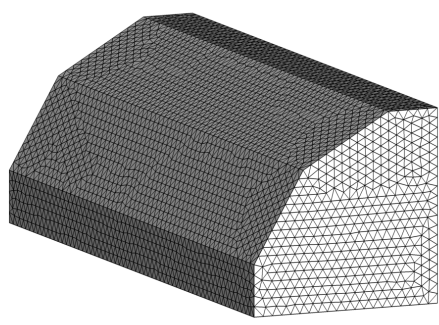

(a) Core surface grid.

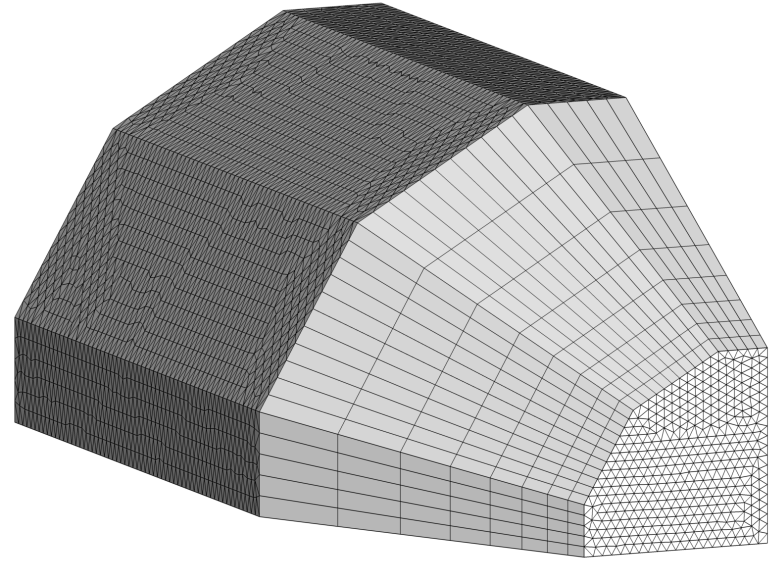

(b) Core and collar inflated surface grid.

Figure 13. Surface grid of a quarter of a cone-cylinder model domain.

\section{First AIAA Sonic Boom Prediction Workshop Grids}

A series of uniformly refined mixed-element and purely-tetrahedral unstructured grids is developed for SBPW-1 with the following considerations: It is expected that the participants would use both cell-centered and node-centered finite volume methods, which have different resolution requirements. ${ }^{60}$ The AIAA Drag Prediction Workshop series emphasized the benefit of a grid convergence study in interpreting results. ${ }^{61}$ Some methods require purely-tetrahedral grids and other methods prefer mixed-element grids. Determining impact of grid element types is also of interest. These considerations are addressed by a series of grids with consistent spacing distributions and common node locations between mixed-element and purely-tetrahedral grids. The Inflate method is used to create the required unstructured mixed-element and purely-tetrahedral grids for SBPW-1. 


\section{V.A. SEEB-ALR Model}

The SEEB-ALR model is described by Morgenstern et al. ${ }^{4}$ It is designed as an axisymmetric body, but a very slight asymmetry was introduced during wind tunnel model construction. The SEEB-ALR solid model is constructed in NX ${ }^{\circledR}$ from a STEP file of the scanned as-built wind tunnel model. A 36-sided prism with regular polygonal bases is created by constructive solid geometry via a batch mode of the GridEx framework. ${ }^{62}$ This prism is as small as possible while containing the SEEB-ALR model to minimize the use of isotropic grids that are not aligned to flow characteristics. The solid model of the SEEB-ALR is Boolean subtracted from the constructed prism. The triangular surface and tetrahedral volume grid are constructed in GridEx using the VGRID size specification ${ }^{17}$ and the FELISA mesher. ${ }^{14,15}$

The surface grid after inflation is shown in Fig. 14(a). The rows of quadrilaterals in Fig. 14 are the sides of prisms that result by extruding the outer triangular boundary of the core grid into prisms. This is the coarsest grid $h=2.0$ in the uniformly refined series and there is only one prism cell for each planar surface in Fig. 14(a). The configuration symmetry plane is shown in Fig. 14(b). The extrusion direction is specified by the Mach number to align to the freestream Mach angle and the layer thickness grows geometrically, Eq. (2). The thickness of the first extruded layer is intended to approximately match the size of adjacent tetrahedra. The core grid is isotropic with fine resolution at the nose, see Fig. 14(b).

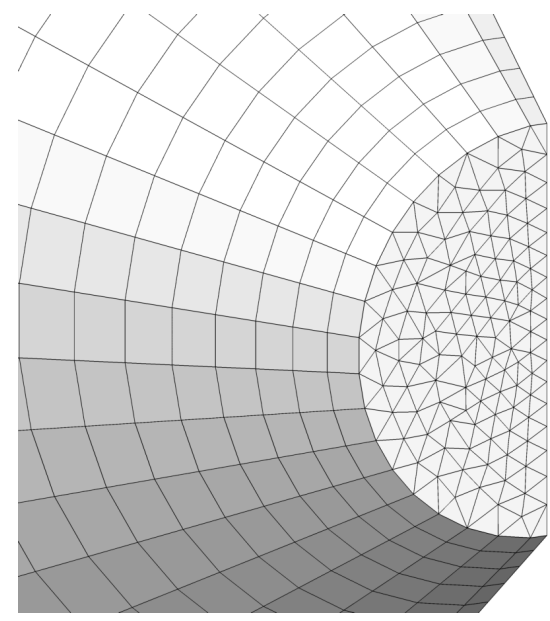

(a) Surface grid for the inflated grid, $h=2.0$.

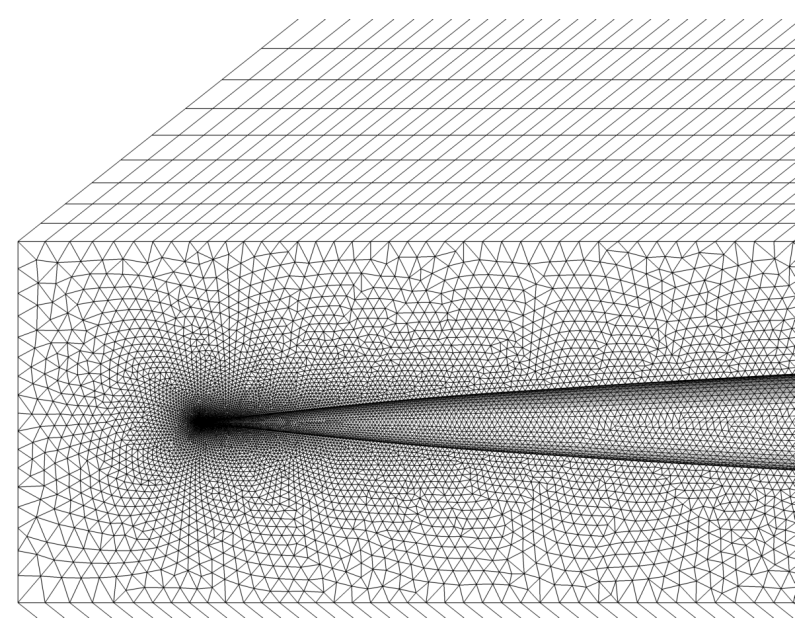

(b) Inflated grid.

Figure 14. SEEB-ALR model with inflated patch topology and surface grid.

A family of uniformly refined grids was developed for the workshop and these grids are required cases. ${ }^{10}$ The total thickness of the inflation is $T=60$ inches. The VGRID spacing field of the inner grid is multiplied by $h$ and a series of grids are produced as listed in Table 1 with the inflation parameters. The $r$ is computed to have a $T=60$ as the number of layers $n$ is varied by inverting Eq. (3). The complete generated grid size of the mixed-element grid is listed in Table 2. The prisms of the mixed-element grids are subdivided into tetrahedra ${ }^{63}$ to form the purely-tetrahedral grids, Table 3 .

Table 1. SEEB-ALR inflation parameters.

\begin{tabular}{lllr} 
Characteristic $h$ & $t_{0}$ & $r$ & $n$ \\
\hline 0.80 & 0.04 & 1.031 & 125 \\
1.00 & 0.05 & 1.040 & 100 \\
1.25 & 0.0625 & 1.050 & 80 \\
1.56 & 0.078 & 1.063 & 64 \\
2.00 & 0.1 & 1.081 & 50
\end{tabular}


Table 2. SEEB-ALR mixed-element grids.

\begin{tabular}{lrrrrr} 
Characteristic $h$ & Nodes & Triangles & Quadrilaterals & Tetrahedra & Prisms \\
\hline 0.80 & $10,159,421$ & 430,082 & 193,875 & $12,015,051$ & $15,850,125$ \\
1.00 & $5,252,466$ & 276,490 & 123,100 & $6,164,094$ & $8,161,100$ \\
1.25 & $2,537,977$ & 172,098 & 80,080 & $3,226,983$ & $3,822,800$ \\
1.56 & $1,165,851$ & 106,222 & 50,240 & $1,614,796$ & $1,682,496$ \\
2.00 & 674,734 & 68,786 & 31,250 & 770,199 & $1,023,350$
\end{tabular}

Table 3. SEEB-ALR purely-tetrahedral grids.

\begin{tabular}{lrrr} 
Characteristic $h$ & Nodes & Triangles & Tetrahedra \\
\hline 0.80 & $10,159,421$ & 817,832 & $59,565,426$ \\
1.00 & $5,252,466$ & 522,690 & $30,647,394$ \\
1.25 & $2,537,977$ & 332,258 & $14,695,383$ \\
1.56 & $1,165,851$ & 206,702 & $6,662,284$ \\
2.00 & 674,734 & 131,286 & $3,840,249$
\end{tabular}

\section{V.B. Delta Wing Body Model}

Originally described as Model 4 in Hunton, Hicks, and Mendoza, ${ }^{64}$ the solid model of the Delta Wing Body is extracted from a STEP file exported from CATIA ${ }^{\circledR}$ V5, where it was created from the description in the original report. The sting was created from drawings used to machine the wind tunnel sting. A simplification was made by omitting the strain gauges, wires, and instrumentation slots visible in a photograph of the model, see Fig. 15. The sting was inserted 0.079184 meters (3.1175 inches) into the wing body and a Boolean addition produced the solid model.

The core region extends well beyond the fuselage to encompass the Delta Wing Body wing, see Fig. 17. A variation in triangle size can be in Fig. 17(b) for the $h=2.0$ outer core grid surface. The leading edge of the extrusion planes are three prisms wide for most planes, see Fig. 18(b). The symmetry plane grid is shown in Fig. 19. The core grid is isotropic and randomly oriented, see Fig. 19(a). The initial layer thickness is twice the adjacent core grid spacing, see Fig. 19(b). The grid parameters are listed in Table 4. The portion of the mixed-element grid that is prismatic is smaller for the Delta Wing Body than the SEEB-ALR, see Table 2 and Table 5.

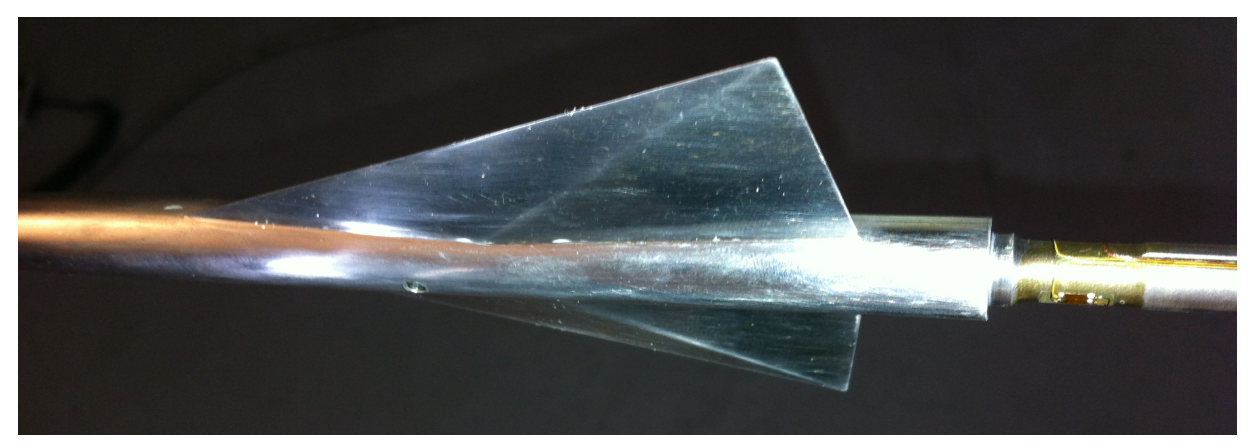

Figure 15. Delta Wing Body and sting.

\section{Signatures}

A flow solution is computed on the grids generated by the three methods and pressure signatures are extracted. USM3D ${ }^{18,19}$ is applied to the BG and MCAP grids. The FUN3D ${ }^{34,35}$ flow solver is applied to the Inflate grids; the van Leer ${ }^{56}$ flux is used with van Albada limiter ${ }^{65}$ for this Euler solution. Examining the signatures from the uniformly refined mixed-element Inflate grids are intended to demonstrate grid 


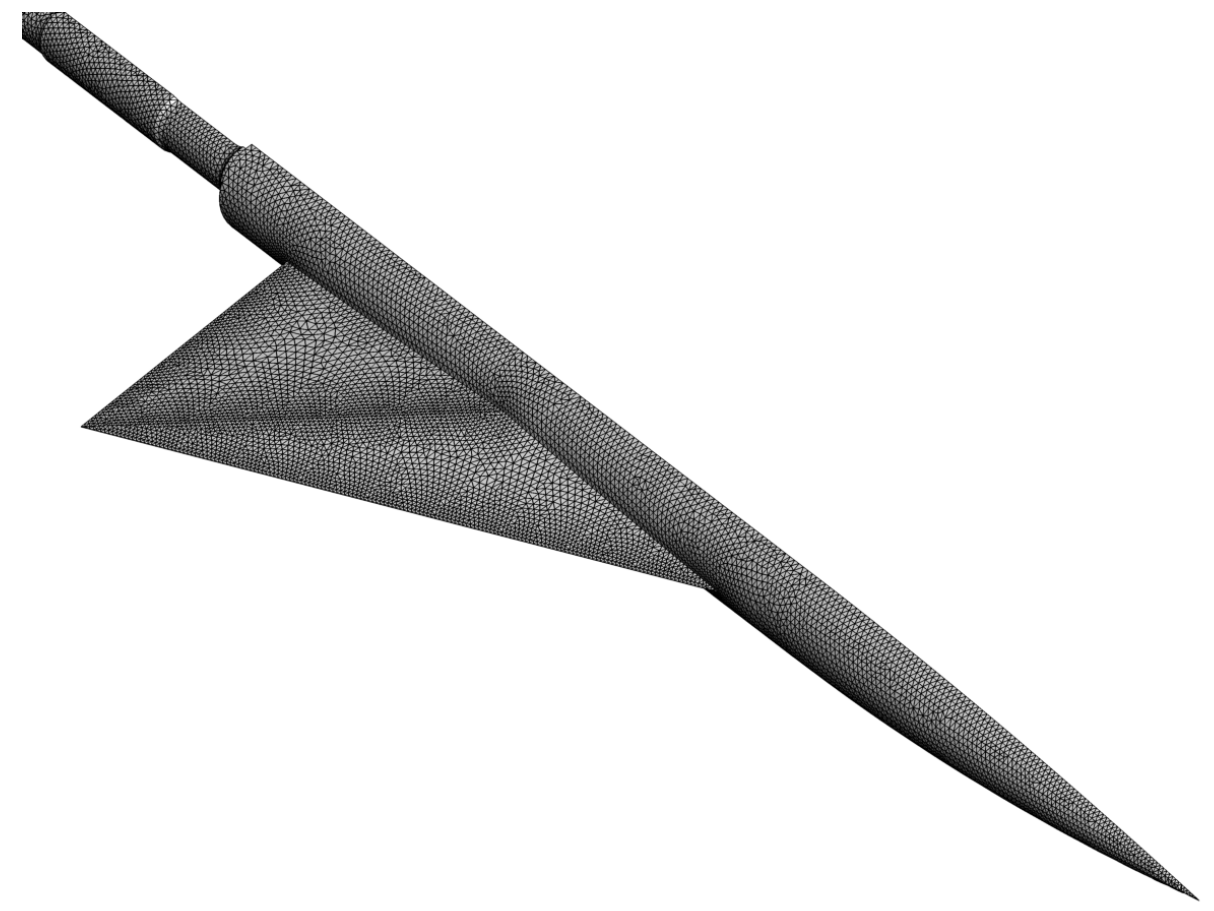

Figure 16. Delta Wing Body surface, $h=2.0$.

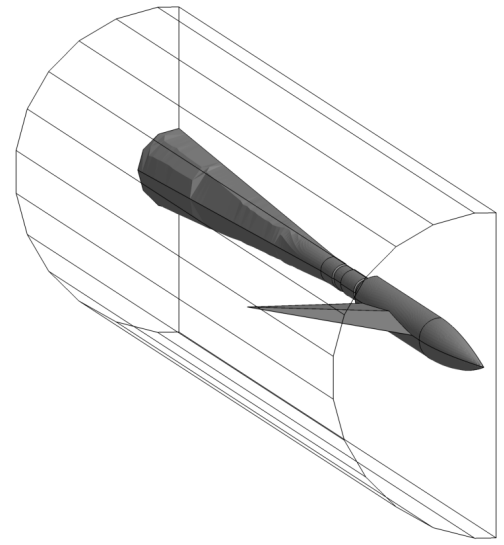

(a) Delta Wing Body model and polygon topology.

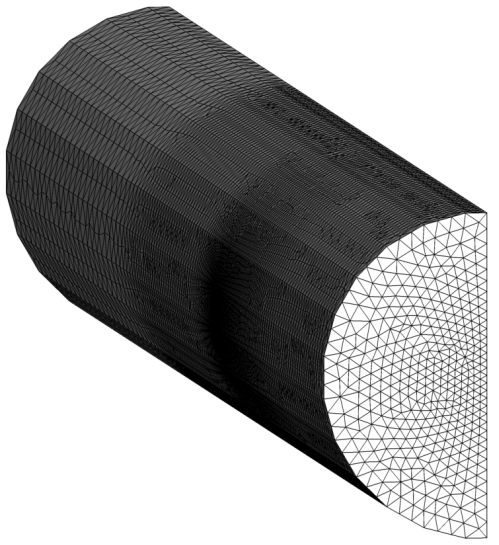

(b) Regular polygon triangular surface, $h=2.0$.

Figure 17. Delta Wing Body model with regular polygon patch topology and surface grid.

Table 4. Delta Wing Body inflation parameters.

\begin{tabular}{lllr} 
Characteristic $h$ & $t_{0}$ & $r$ & $n$ \\
\hline 1.00 & 0.002500 & 1.022 & 100 \\
1.25 & 0.003125 & 1.028 & 80 \\
1.56 & 0.003900 & 1.035 & 64 \\
2.00 & 0.005000 & 1.045 & 50
\end{tabular}




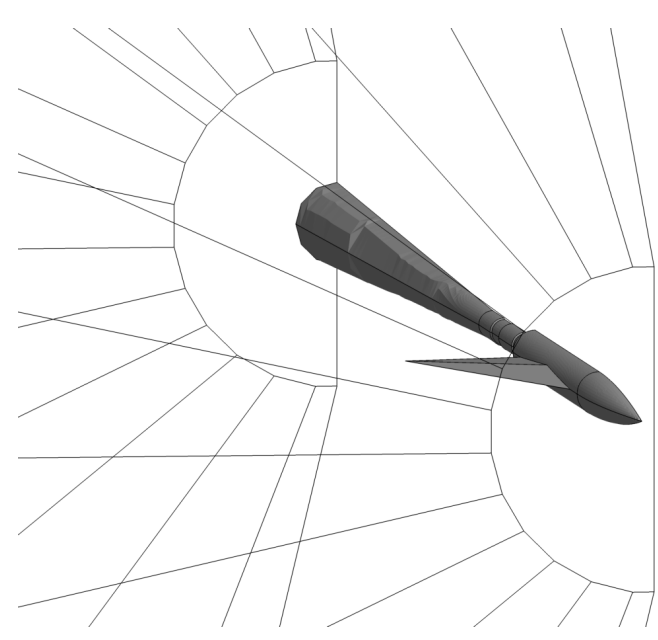

(a) Delta Wing Body model and inflated grid topology.

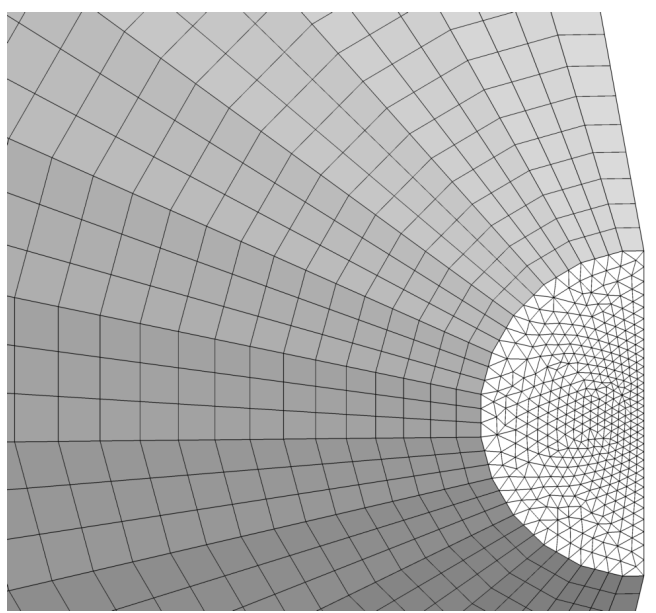

(b) Surface grid for the inflated grid, $h=2.0$.

Figure 18. Delta Wing Body model with inflated patch topology and surface grid.

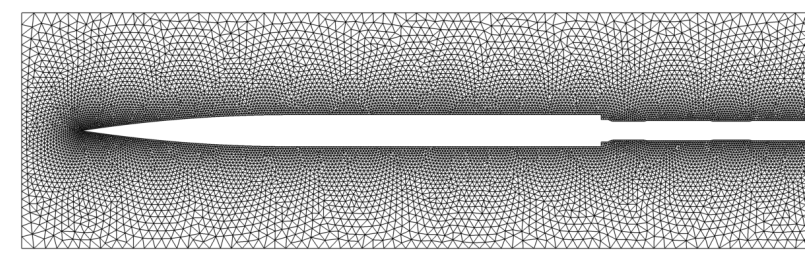

(a) Inner grid.

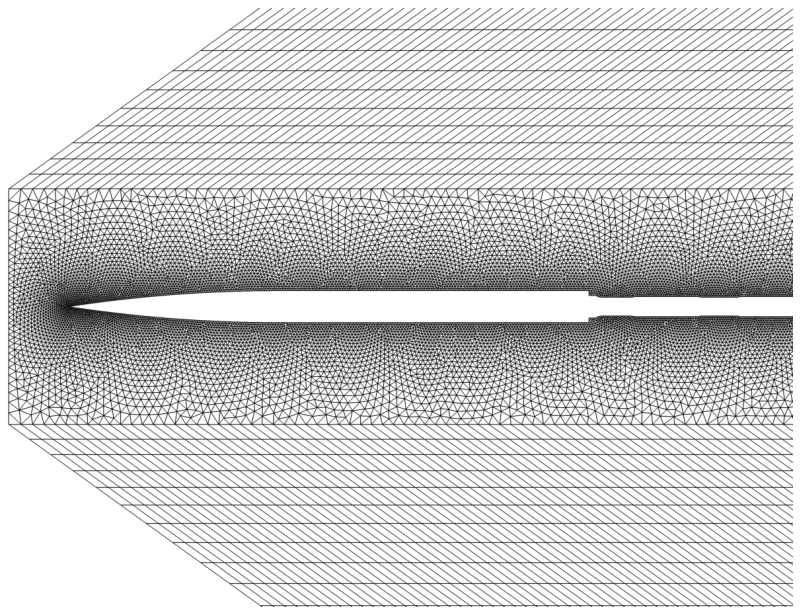

(b) Inflated grid.

Figure 19. Delta Wing Body model surface grid with the symmetry plane of the original and inflated grids.

Table 5. Delta Wing Body mixed-element grids.

\begin{tabular}{lrrrrr} 
Characteristic $h$ & Nodes & Triangles & Quadrilaterals & Tetrahedra & Prisms \\
\hline 1.00 & $5,363,065$ & 249,640 & 55,000 & $17,734,283$ & $4,689,000$ \\
1.25 & $2,742,341$ & 159,652 & 34,880 & $9,081,579$ & $2,369,920$ \\
1.56 & $1,411,515$ & 102,688 & 22,464 & $4,671,872$ & $1,205,440$ \\
2.00 & 695,709 & 63,226 & 13,700 & $2,217,575$ & 613,000
\end{tabular}


Table 6. Delta Wing Body purely-tetrahedral grids.

\begin{tabular}{lrrr} 
Characteristic $h$ & Nodes & Triangles & Tetrahedra \\
\hline 1.00 & $5,363,065$ & 359,640 & $31,801,283$ \\
1.25 & $2,742,341$ & 229,412 & $16,191,339$ \\
1.56 & $1,411,515$ & 147,616 & $8,288,192$ \\
2.00 & 695,709 & 90,626 & $4,056,575$
\end{tabular}

convergence. The finest mixed-element Inflate, finest purely-tetrahedra Inflate, purely-tetrahedra BG and purely-tetrahedra MCAP are combined with experimental data to illustrate the properties of each method.

\section{VI.A. SEEB-ALR Signature}

FUN3D ${ }^{34,35}$ is applied uniformly refined mixed-element Inflate grids for the SEEB-ALR. The signature at $H=21.2$ inches is shown in Fig. 20. All five mixed-element grids are shown in Fig. 20(a). The similarity for the signatures from these series of refined grids indicates that grids are sufficiently resolved. Only a slight increase in sharpness at the extrema is observed.

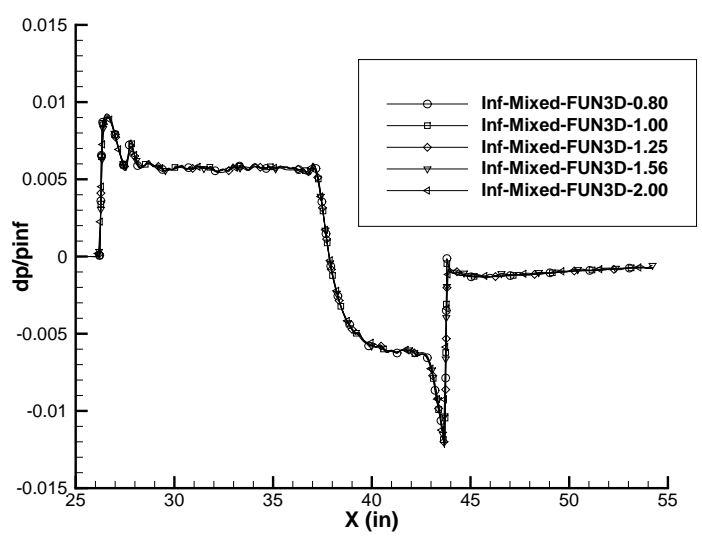

(a) Uniformly refined mixed-element grids

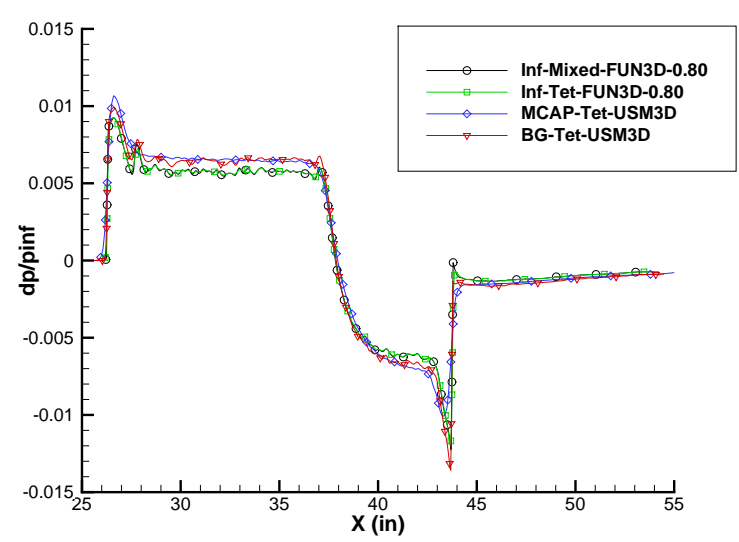

(b) Various grid methods.

Figure 20. SEEB-ALR signatures at $H=21.2$ inches

The MCAP and BG grid signatures are plotted with the finest $h=0.80$ mixed-element and the finest purely-tetrahedral grid signatures in Fig. 20(b). The purely-tetrahedral and mixed-element Inflate grid signatures over plot on this scale. The MCAP-Tet-USM3D and BG-Tet-USM3D also produce similar signatures with a higher pressure flat-top and lower pressure expansion than the Inflate grids. The reason for this slight difference is unknown. The model surface grids are inspected to eliminate geometry errors as a source of the difference. The amount of difference between the signatures is placed in context by comparing it to the magnitude of the experimental uncertainty.

The sonic boom pressure signatures were measured in the NASA Ames 9- by 7-Foot Supersonic Wind Tunnel at Mach 1.6 and 1.7 in 2011 and 2012 during phase I and II of the NASA High Speed Project $\mathrm{N}+2$ Experimental Systems Validations NASA Research Announcement (NRA) contract. ${ }^{4}$ The sonic boom test data were obtained using pressure rail instrumentation developed by NASA in $2010 .{ }^{66}$ The rail design effectively eliminates amplification or reflection of the shock wave strength at pressure measuring orifices on the tip of rail, and is designated the reflection factor 1.0 (RF1) rail. Spatially averaged pressure signatures measured at multiple models positions along the RF1 rail were used to provide the accurate sonic test data. ${ }^{67}$ Additional description of the experimental data for a variety of flow conditions, including the data near the flow conditions of the SBPW-1 test cases, are provided in a companion paper. ${ }^{68}$

The computed signatures are compared with spatially-averaged experimental measurements from two sets of wind tunnel test runs in Fig. 21. All methods are within the experimental uncertainty for the flat-top 
portion of the forward signature $x=[28,37]$ and below the experimental expansion pressure $x=[38,43]$ including uncertainty. The final recovery pressure $x=[45,55]$ is within the uncertainty of the experimental measurements.

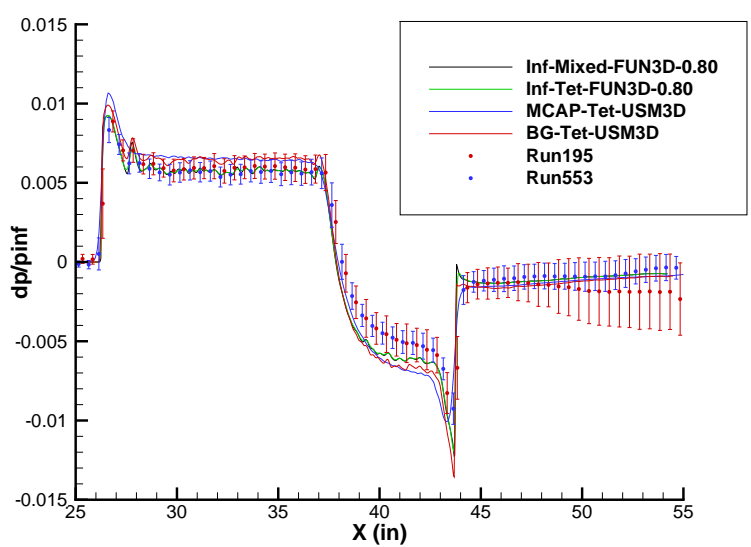

Figure 21. SEEB-ALR signatures at $H=21.2$ inches, various grid methods and experimental data. ${ }^{68}$

\section{VI.B. Delta Wing Body Signature}

The SEEB-ALR is an axisymmetric body (with a small eccentricity due to as-built tolerances). The Delta Wing Body has axisymmetric body with a wing, so it provides axisymmetric signatures forward of the wing and with varying off-track signatures aft of the wing for evaluating the suitability of grids for off-track propagation. The signature at $H=0.5$ inches below the model is shown in Fig. 22. All four mixed-element grids are shown in Fig. 22(a). The signature changes little with grid refinement in the forward portion of the signature. The model base region $x=[0.18,0.28]$ changes the most with grid refinement and the faceting of the sting surface grid is evident for the coarser grids aft of $x=0.28$.

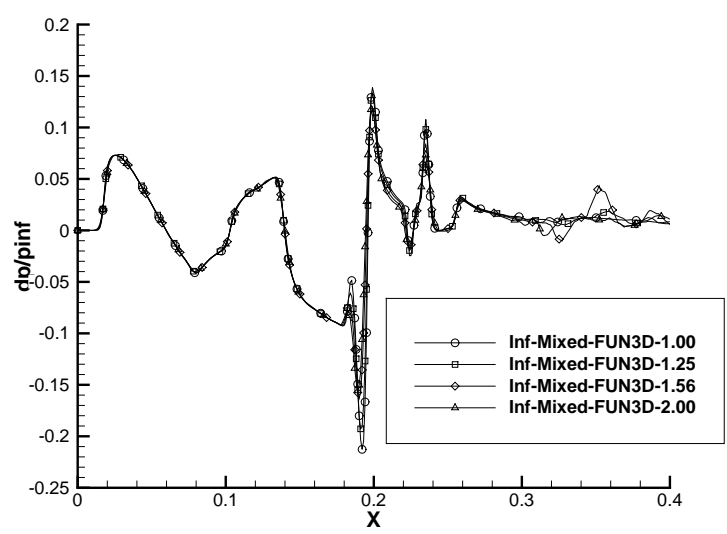

(a) Uniformly refined mixed-element grids.

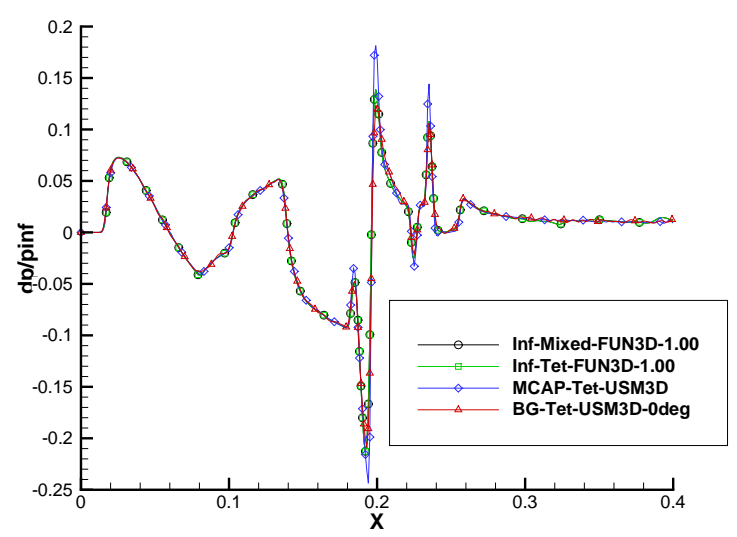

(b) Various grid methods.

Figure 22. Delta Wing Body signatures at $H=0.5$ and $\phi=0$.

The BG grid has clustering in the $\phi=0$ direction as is shown in Fig. 7(a). The finest $h=1.00$ mixedelement Inflate grid signature is shown with the finest purely-tetrahedral Inflate grid signature in Fig. 22(b). The purely-tetrahedral and mixed-element Inflate grid signatures are identical because the grids are identical within the core region. The signature are effectively identical for all methods at this close distance, centerline location, except for the extrema. 
The signature at $H=31.8$ inches and $\phi=30$ is shown in Fig. 23. All four mixed-element grids are shown in Fig. 23(a). The signature forward of $x=1.25$ remains similar during grid refinement. The signature influenced by the model base region $x=[1.28,1.35]$ changes the most with grid refinement and faceting of the sting surface grid aft of $x=1.35$ is still evident at this propagation distance.

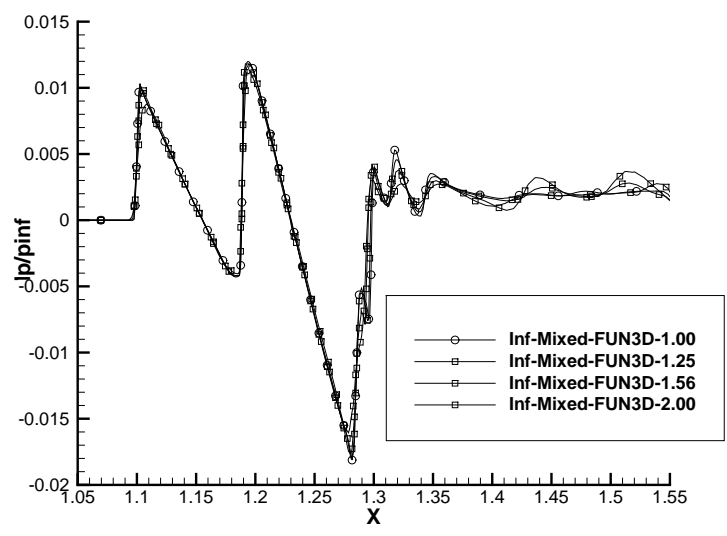

(a) Uniformly refined mixed-element grids.

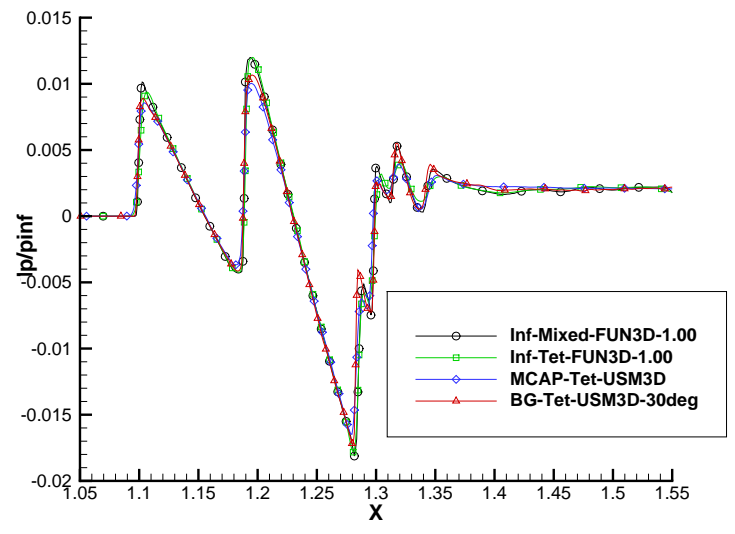

(b) Various grid methods.

Figure 23. Delta Wing Body signatures at $H=31.8$ and $\phi=30$.

The finest $h=1.00$ mixed-element Inflate grid signature is shown with the finest purely-tetrahedral Inflate grid signature in Fig. 23(b). The BG grid has clustering in the $\phi=30$ direction. An example of this off-track clustering is shown in Fig. 7(b). The purely-tetrahedral and mixed-element signatures are similar with a slight smoothing at $x=1.32$ for the Inf-Tet-FUN3D-1.00 and MCAP-Tet-USM3D signatures.

The signature at $H=31.8$ inches and $\phi=60$ is shown in Fig. 24. All four mixed-element grids are shown in Fig. 24(a). The signature forward of $x=1.25$ remains similar during grid refinement. The signature influenced by the model base region $x=[1.27,1.35]$ changes the most with grid refinement and faceting of the sting surface grid aft of $x=1.35$ is still evident at this propagation distance.

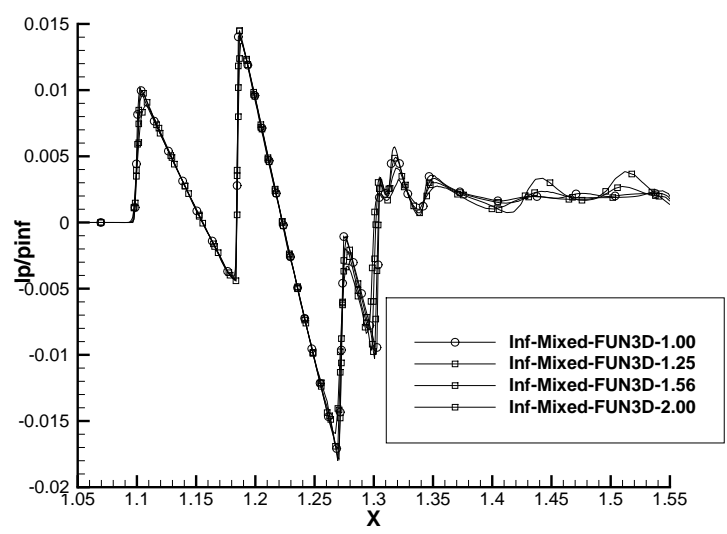

(a) Uniformly refined mixed-element grids.

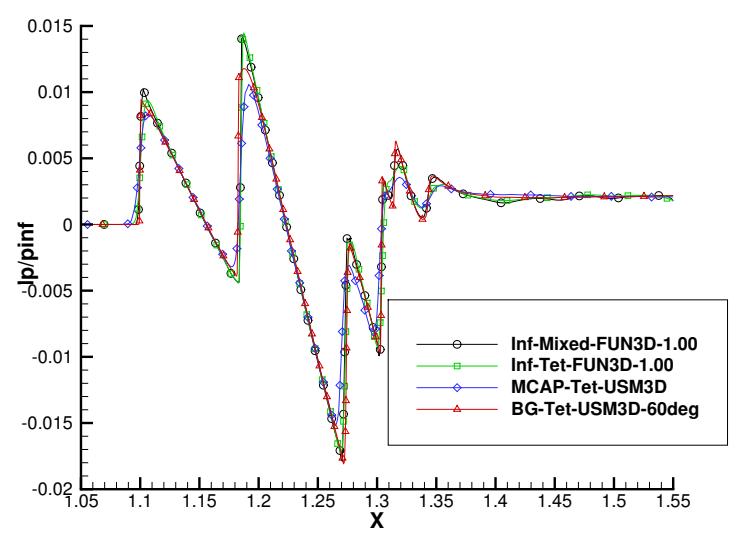

(b) Various grid methods.

Figure 24. Delta Wing Body signatures at $H=31.8$ and $\phi=60$.

The finest $h=1.00$ mixed-element Inflate grid signature is shown with the finest purely-tetrahedral Inflate grid signature in Fig. 24(b). The purely-tetrahedral Inflate, mixed-element Inflate, and MCAP signatures are similar with a slight smoothing at $x=1.32$ for the purely-tetrahedral Inflate and MCAP signatures. The BG grid has clustering in the $\phi=60$ direction, which is enabled by the COB improvement to the core grid outer surface. A typical vehicle boom carpets is less then 60 degrees, ${ }^{4}$ which makes the $\phi=60$ extraction location uncommon in practice and a robustness test of the grid generation methods. 


\section{Conclusions}

A review of published methods for specialized near-field sonic boom prediction grid generation methods is provided. The influence of uniform grid refinement is studied for a slight ( 4 degrees) misalignment of the grid, where refinement in the propagation direction is more effective than streamwise refinement for reducing rounding of the signature. The freestream Mach angle aligned grids exhibited the expected sawtooth wave. This study emphasizes the importance of aligning the grid stretching with shocks and expansions for sonic boom propagation. Splitting the hexahedral cells of the 3D example into prismatic cells increased the rounding of misaligned grids.

Issues related to the robust extrusion of triangulated cylindrical surfaces is detailed and a description of three collar grid extrusion techniques based on tetrahedral core grids is provided. Each method addresses the robustness of collar grid extrusion differently. The three methods are applied to models from the First AIAA Sonic Boom Workshop to illustrate the utility of these methods. Uniform grid refinement showed grid convergence properties of the Inflate method for the SEEB-ALR and Delta Wing Body. These uniformly refined grids are required unstructured grids for the workshop. The differences between the signatures extracted from the various grid generation methods was equivalent to the magnitude of the experimental uncertainty.

\section{Acknowledgments}

Hiroaki Nishikawa, Bil Kleb, and Boris Diskin provided useful discussions on the alignment of grids to shocks. Yoshikazu Makino provided the solid model of the Delta Wing Body and Bil Kleb created the Delta Wing Body sting model.

\section{References}

${ }^{1}$ Henne, P. A., "Case for Small Supersonic Civil Aircraft," AIAA Journal of Aircraft, Vol. 42, No. 3, May-June 2005, pp. 765-774.

${ }^{2}$ Sakata, K., "Japan's Supersonic Technology and Business Jet Perspectives," AIAA Paper 2013-21, 2013.

${ }^{3}$ Magee, T. E., Wilcox, P. A., Fugal, S. R., Acheson, K. E., Adamson, E. E., Bidwell, A. L., and Shaw, S. G., "SystemLevel Experimental Validations for Supersonic Commercial Transport Aircraft Entering Service in the 2018-2020 Time Period," NASA CR-2013-217797, NASA Langley Research Center, Feb. 2013.

${ }^{4}$ Morgenstern, J., Norstrud, N., Sokhey, J., Martens, S., and Alonso, J. J., "Advanced Concept Studies for Supersonic Commercial Transports Entering Service in the 2018 to 2020 Period," NASA CR-2013-217820, NASA Langley Research Center, Feb. 2013.

${ }^{5}$ Bedard, Jr., A. J., "The Measurement of Sonic Boom Waveforms and Propagation Characteristics: Techniques and Challenges," AIAA Paper 90-4004, 1990.

${ }^{6}$ Plotkin, K. J., "State of the Art of Sonic Boom Modeling," Journal of the Acoustical Society of America, Vol. 111, No. 1, Jan. 2002, pp. 530-536.

${ }^{7}$ Ozcer, I. A., Sonic Boom Prediction Using Euler/Full-Potential Methodology, Master's thesis, Old Dominion University, Dec. 2005.

${ }^{8}$ Park, M. A., Anisotropic Output-Based Adaptation with Tetrahedral Cut Cells for Compressible Flows, Ph.D. thesis, Massachusetts Institute of Technology, Sept. 2008.

${ }^{9}$ Park, M. A., Aftosmis, M. J., Campbell, R. L., Carter, M. B., Cliff, S. E., and Bangert, L. S., "Summary of the 2008 NASA Fundamental Aeronautics Program Sonic Boom Prediction Workshop," AIAA Paper 2013-649, 2013.

${ }^{10}$ http://lbpw.larc.nasa.gov [cited 7 November 2013].

${ }^{11}$ Waithe, K. A., "Introduction of First Low Boom Prediction Workshop," AIAA Paper 2013-650, 2013.

${ }^{12}$ Cliff, S. E. and Thomas, S. D., "Euler/Experiment Correlations of Sonic Boom Pressure Signatures," AIAA Journal of Aircraft, Vol. 30, No. 5, Sept.-Oct. 1993, pp. 669-675.

${ }^{13}$ Djomehri, M. J. and Erickson, L. L., "An Assessment of the Adaptive Unstructured Tetrahedral Grid, Euler Flow Solver Code FELISA," NASA TP-3526, NASA Ames Research Center, Dec. 1994.

${ }^{14}$ Peraire, J., Peirò, J., and Morgan, K., "Adaptive Remeshing for Three-Dimensional Compressible Flow Computations," Journal of Computational Physics, Vol. 103, No. 2, 1992, pp. 269-285.

${ }^{15}$ Peiró, J., Peraire, J., and Morgan, K., "FELISA System Version 1.1 Reference Manual Part 2 - User Manual," University of Wales/Swansea Technical Report CR/822/94, Aug. 1994

${ }^{16}$ Fouladi, K., "Unstructured Grids for Sonic-Boom Analysis," AIAA Paper 99-2929, 1993.

${ }^{17}$ Pirzadeh, S. Z., "Structured Background Grids for Generation of Unstructured Grids by Advancing-Front Method," AIAA Journal, Vol. 31, No. 2, Feb. 1993, pp. 257-265.

${ }^{18}$ Frink, N. T., "Upwind scheme for solving the Euler equations on unstructured tetrahedral meshes," AIAA Journal, Vol. 30, No. 1, Jan. 1992, pp. 70-77. 
${ }^{19}$ Frink, N. T., Three-Dimensional Upwind Scheme for Solving the Euler Equations on Unstructured Tetrahedral Grids, Ph.D. thesis, Virginia Polytechnic Institute and State University, Sept. 1991.

${ }^{20}$ Choi, S., Alonso, J. J., and van der Weide, E., "Numerical and Mesh Resolution Requirements for Accurate Sonic Boom Prediction," AIAA Journal of Aircraft, Vol. 46, No. 4, July-Aug. 2009, pp. 1126-1139.

${ }^{21}$ Carter, M. and Deere, K., "Grid Sourcing and Adaptation Study Using Unstructured Grids for Supersonic Boom Prediction," AIAA Paper 2008-6595, 2008.

${ }^{22}$ Campbell, R., Carter, M., Deere, K., and Waithe, K. A., "Efficient Unstructured Grid Adaptation Methods for Sonic Boom Prediction," AIAA Paper 2008-7327, 2008.

${ }^{23}$ Carter, M. B., Campbell, R. L., and Nayani, S. N., "USM3D Analysis of Low Boom Configuration," AIAA Paper 2011-3335, 2011.

${ }^{24}$ Nayani, S. N. and Campbell, R. L., "Evaluation of Grid Modification Methods for On- and Off-Track Sonic Boom Analysis," AIAA Paper 2013-798, 2013.

${ }^{25}$ Cliff, S. E., Elmiligui, A. A., Campbell, R. L., and Thomas, S. D., "Evaluation of Refined Tetrahedral Meshes with Projected, Stretched, and Sheared Prism Layers for Sonic Boom Analysis," AIAA Paper 2011-3338, 2011.

${ }^{26}$ Meredith, K., Dahlin, J., Graham, D., Malone, M., Haering, E., Page, J., and Plotkin, K., "Computational Fluid Dynamics Comparison and Flight Test Measurement of F-5E Off-Body Pressures," AIAA Paper 2005-6, 2005.

${ }^{27}$ Bui, T. T., "Computational Fluid Dynamics Analysis of Nozzle Plume Effects on Sonic Boom Signature," AIAA Journal of Aircraft, Vol. 48, No. 2, March-April 2011, pp. 368-380.

${ }^{28}$ Ishikawa, H., Makino, Y., Ito, T., and Kuroda, F., "Sonic Boom Prediction Using Multi-Block Structured Grids CFD Code Considering Jet-On Effects," AIAA Paper 2009-3508, 2009.

${ }^{29}$ Siclari, M. J. and Darde, C. M., "An Euler Code Prediction of Near-Field to Midfield Sonic Boom Pressure Signatures," AIAA Journal of Aircraft, Vol. 30, No. 6, Nov.-Dec. 1993, pp. 911-917.

${ }^{30}$ Page, J. A. and Plotkin, K. J., "An Efficient Method for Incorporating Computational Fluid Dynamics into Sonic Boom Prediction," AIAA Paper 91-3275, 1991.

${ }^{31}$ Kandil, O. A., Yang, Z., and Bobbitt, P. J., "Prediction of Sonic Boom Signature Using Euler-Full Potential CFD with Grid Adaptation and Shock Fitting," AIAA Paper 2002-2542, 2002.

${ }^{32}$ Kandil, O. and Ozcer, I. A., "Sonic Boom Computations for Double-Cone Configuration using CFL3D, FUN3D and Full-Potential Codes," AIAA Paper 2006-414, 2006.

${ }^{33}$ Ozcer, I. A. and Kandil, O., "FUN3D / OptiGRID Coupling for Unstructured Grid Adaptation for Sonic Boom Problems," AIAA Paper 2008-61, 2008.

${ }^{34}$ Anderson, W. K. and Bonhaus, D. L., "An Implicit Upwind Algorithm for Computing Turbulent Flows on Unstructured Grids," Computers and Fluids, Vol. 23, No. 1, 1994, pp. 1-22.

${ }^{35}$ Nielsen, E. J., Aerodynamic Design Sensitivities on an Unstructured Mesh Using the Navier-Stokes Equations and a Discrete Adjoint Formulation, Ph.D. thesis, Virginia Polytechnic Institute and State University, 1998.

${ }^{36}$ Suerich-Gulick, F., Lepage, C. Y., and Habashi, W. G., "Anisotropic 3-D Mesh Adaptation on Unstructured Hybrid Meshes," AIAA Paper 2002-859, 2002.

${ }^{37}$ Alauzet, F. and Loseille, A., "High-Order Sonic Boom Modeling Based on Adaptive Methods," Journal of Computational Physics, Vol. 229, No. 3, 2010, pp. 561-593.

${ }^{38}$ Loseille, A., Dervieux, A., and Alauzet, F., "Fully Anisotropic Goal-Oriented Mesh Adaptation for 3D Steady Euler Equations," Journal of Computational Physics, Vol. 229, No. 8, 2010, pp. 2866-2897.

${ }^{39}$ Jones, W. T., Nielsen, E. J., and Park, M. A., "Validation of 3D Adjoint Based Error Estimation and Mesh Adaptation for Sonic Boom Prediction," AIAA Paper 2006-1150, 2006.

${ }^{40}$ Park, M. A., "Low Boom Configuration Analysis with FUN3D Adjoint Simulation Framework," AIAA Paper 2011-3337, 2011.

${ }^{41}$ Barter, G. E., Shock Capturing with PDE-Based Artificial Viscosity for an Adaptive, Higher-Order Discontinuous Galerkin Finite Element Method, Ph.D. thesis, Massachusetts Institute of Technology, 2008.

${ }^{42}$ Fidkowski, K. J. and Darmofal, D. L., "Output-Based Adaptive Meshing Using Triangular Cut Cells," Technical Report ACDL TR-06-2, Aerospace Computational Design Laboratory, Department of Aeronautics and Astronautics, Massachusetts Institute of Technology, 2006.

${ }^{43}$ Nemec, M., Aftosmis, M. J., and Wintzer, M., "Adjoint-Based Adaptive Mesh Refinement for Complex Geometries," AIAA Paper 2008-725, 2008.

${ }^{44}$ Aftosmis, M. J., Berger, M. J., and Adomavicius, G., "A Parallel Multilevel Method for Adaptively Refined Cartesian Grids with Embedded Boundaries," AIAA Paper 2000-808, 2000.

${ }^{45}$ Cliff, S. E., Thomas, S. D., McMullen, M. S., Melton, J. E., and Durston, D. A., "Assessment of Unstructured Euler Methods for Sonic Boom Pressure Signatures Using Grid Refinement and Domain Rotation Methods," NASA TM-2008-214568, Sept. 2008.

${ }^{46}$ Aftosmis, M. J., Nemec, M., and Cliff, S. E., "Adjoint-Based Low-Boom Design with Cart3D," AIAA Paper 2011-3500, 2011.

${ }^{47}$ Alonso, J. J., Kroo, I. M., and Jameson, A., "Advanced Algorithms for Design and Optimization of Quiet Supersonic Platforms," AIAA Paper 2002-144, 2002.

${ }^{48}$ Laflin, K. R., Klausmeyer, S. M., and Chaffin, M., "A Hybrid Computational Fluid Dynamics Procedure for Sonic Boom Prediction," AIAA Paper 2006-3168, 2006.

${ }^{49}$ Mavriplis, D. J., "Viscous Flow Analysis Using a Parallel Unstructured Multigrid Solver," AIAA Journal, Vol. 38, No. 11, Nov. 2000, pp. 2067-2076.

${ }^{50}$ Krist, S. L., Biedron, R. T., and Rumsey, C. L., "CFL3D User's Manual (Version 5.0)," NASA TM-208444, NASA Langley Research Center, June 1998. 
${ }^{51}$ Waithe, K. A., "Application of USM3D for Sonic Boom Prediction by Utilizing a Hybrid Procedure," AIAA Paper 2008-129, 2008.

${ }^{52}$ Nichols, R. H., Tramel, R. W., and Buning, P. G., "Solver and Turbulence Model Upgrades to OVERFLOW 2 for Unsteady and High-Speed Applications," , No. 2006-2824, 2006.

${ }^{53}$ Howe, D. C., "Hybrid CART3D/OVERFLOW Near-Field Analysis of a Low Boom Configuration with Wind Tunnel Comparisons," AIAA Paper 2011-3336, 2011.

${ }^{54}$ Ishikawa, H., Tanaka, K., Makino, Y., and Yamamoto, K., "Sonic-Boom Prediction Using Euler CFD Codes with Structured/Unstructured Overset Method," 27th International Congress of the Aeronautical Sciences (ICAS), 2010.

${ }^{55}$ Morgenstern, J. M., Buonanno, M., and Marconi, F., "Full Configuration Low Boom Model and Grids for 2014 Sonic Boom Prediction Workshop," AIAA Paper 2013-647, 2013.

${ }^{56}$ van Leer, B., "Flux-Vector Splitting for the Euler Equations," Lecture Notes in Physics, Vol. 170, 1982, pp. 507-512.

${ }^{57}$ Shewchuk, J. R., "What Is a Good Linear Element? Interpolation, Conditioning, and Quality Measures," 11th International Meshing Roundtable, Sept. 2002.

${ }^{58} \mathrm{Li}$, W., Campbell, R., Geiselhart, K., Shields, E., Nayani, S., and Shenoy, R., "Integration of Engine, Plume, and CFD Analyses in Conceptual Design of Low-Boom Supersonic Aircraft," AIAA Paper 2009-1171, 2009.

${ }^{59}$ Elmiligui, A. A., Wilcox, F. J., Cliff, S. E., and Thomas, S. D., "Numerical Predictions of Sonic Boom Signatures for a Straight Line Segmented Leading Edge Model," Seventh International Conference on Computational Fluid Dynamics (ICCFD7), July 2012.

${ }^{60}$ Diskin, B. and Thomas, J. L., "Comparison of Node-Centered and Cell-Centered Unstructured Finite-Volume Discretizations: Inviscid Fluxes," AIAA Journal, Vol. 49, No. 4, April 2011, pp. 1326-1338.

${ }^{61}$ Mavriplis, D. J., Vassberg, J. C., Tinoco, E. N., Mani, M., Brodersen, O. P., Eisfeld, B., Wahls, R. A., Morrison, J. H., Zickuhr, T., Levy, D., and Murayama, M., "Grid Quality and Resolution Issues from the Drag Prediction Workshop Series," AIA A Journal of Aircraft, Vol. 46, No. 3, May-June 2009, pp. 935-950.

${ }^{62}$ Jones, W. T., "GridEx - An Integrated Grid Generation Package for CFD," AIAA Paper 2003-4129, 2003.

${ }^{63}$ Dompierre, J., Labbé, P., Vallet, M.-G., and Camarero, R., "How to Subdivide Pyramids, Prisms, and Hexahedra into Tetrahedra," 8th International Meshing Roundtable, 1999.

${ }^{64}$ Hunton, L. W., Hicks, R. M., and Mendoza, J. P., "Some Effects of Wing Planform on Sonic Boom," NASA TN D-7160, NASA Ames Research Center, Jan. 1973.

${ }^{65}$ van Albada, G. D., van Leer, B., and Roberts, Jr., W. W., "A Comparative Study of Computational Methods in Cosmic Gas Dynamics," Astronomy and Astrophysics, Vol. 108, 1982, pp. 76-84.

${ }^{66}$ Cliff, S. E., Elmiligui, A. A., Aftosmis, M. J., Thomas, S. D., Morgenstern, J. M., and Durston, D. A., "Design and Evaluation of a Pressure Rail for Sonic Boom Measurement in Wind Tunnels," Seventh International Conference on Computational Fluid Dynamics (ICCFD7), July 2012.

${ }^{67}$ Morgenstern, J. M., "How to Accurately Measure Low Sonic Boom or Model Surface Pressures in Supersonic Wind Tunnels," AIAA Paper 2012-3215, 2012.

${ }^{68}$ Cliff, S. E., Durston, D. A., Elmiligui, A., Jensen, J. C., and Chan, W. M., "Computation and Experimental Assessment of Models for the First AIAA Sonic Boom Prediction Workshop," AIAA Science and Technology Forum and Exposition (SciTech2014), American Institute of Aeronautics and Astronautics, Reston, VA (submitted for publication). 